NASA Contractor Report 4505

\title{
Roughness-Induced Generation of Crossflow Vortices in Three-Dimensional Boundary Layers
}

Meelan Choudhari

High Technology Corporation

Hampton, Virginia

Prepared for

Langley Research Center

under Contract NAS1-19299

\section{N/SA}

National Aeronautics and

Space Administration

Office of Management

Scientific and Technical

Information Program

1993 



\title{
Roughness-Induced Generation of Crossflow Vortices in Three-Dimensional Boundary Layers
}

\author{
Meelan Choudhari \\ High Technology Corporation \\ Hampton, VA 23666
}

\begin{abstract}
The receptivity theory of Goldstein and Ruban is extended within the nonasymptotic (quasiparallel) framework of Zavol'skii et al to predict the roughness-induced generation of stationary and nonstationary instability waves in three-dimensional, incompressible boundary layers. The influence of acoustic-wave orientation, as well as that of different types of roughness geometries, including isolated roughness elements, periodic arrays and two-dimensional lattices of compact roughness shapes, as well as random, but spatially homogeneous roughness distributions, is examined. The parametric study for the Falkner-Skan-Cooke family of boundary layers supports our earlier conjecture that the initial amplitudes of roughness-induced stationary vortices are likely to be significantly larger than the amplitudes of similarly induced nonstationary vortices in the presence of acoustic disturbances in the free stream. Maximum unsteady receptivity occurs when the acoustic velocity fluctuation is aligned with the wavenumber vector of the unsteady vortex mode. On the other hand, roughness arrays that are oriented somewhere close to the group velocity direction are likely to produce higher instability amplitudes. Limitations of the nonasymptotic theory are discussed, and future work is suggested.
\end{abstract}




\section{Background}

Although most boundary-layer flows of engineering importance are three-dimensional in nature, much of the research related to their transition from the laminar to a turbulent state has focused on twodimensional boundary layers, particularly in view of the complexity of the physical phenomena involved in the transition process. However, continued interest in the utilization of laminar flow technolgy in real-world applications has led to a concerted effort in the recent years, by both theoreticians and experimentalists, in the area of the stability and transition of three-dimensional boundary layers. A competent review of the research published until 1989 was given by Saric and Reed [1], who also provided an extensive bibliography on the subject. Notable progress since then includes the secondary instability analysis for a rotating disk boundary layer by Balachandar et al [2], experiments by Saric and his colleagues on the primary [3] and secondary [4] instabilities, plus the receptivity [5] of a swept-wing boundary layer, the temporal simulation of the transition in a swept-wing boundary layer by Meyer and Kleiser [6], and the initiation of spatial simulations by Reed [7], and Joslin and Streett [8].

The transition in a three-dimensional boundary layer can involve any one or more of the following four types of disturbances: (i) attachment-line instability and/or contamination, (ii) crossflow vortices, which can be either stationary or nonstationary, (iii) streamwise instabilities, including both the viscousand inviscid-type modes, and (iv) the centrifugal, or Görtler, instability. From these, disturbances along the attachment line are of paramount importance in laminar flow technology, because they can lead to a turbulent boundary layer over the entire surface of the wing. However, if and when the attachmentline flow can be maintained subcritical, and its contamination avoided by using techniques such as the Gaster bump [9], the boundary-layer transition on the (typically convex) upper surface of a laminarflow airfoil is initiated by the crossflow vortices that dominate the favorable pressure gradient region

close to the leading edge of this airfoil. Hence, in order to understand and successfully predict the transition process in such flows, the different mechanisms by which these crossflow vortices are excited within the boundary layer must be understood. Transition along the (partly concave) 'lower surface of the airfoil can also involve the centrifugal instabilities; the generation of these instabilities, by virtue of disturbances on the airfoil surface as well as in the free stream, has been described by Hall [10] and Denier, Hall, and Seddougui [11], respectively. The effects of crossflow on the stability and the generation of these modes has been studied by Bassom and Hall [12].

The first definitive work on the crossflow-receptivity problem was carried out by Wilkinson and 
Malik [13], who traced the origin of stationary crossflow vortices in a rotating disk boundary layer to naturally occuring, isolated roughness sites on the disk surface. Subsequently, this finding was verified through controlled experiments that involved artificially introduced roughness sites. The overall conclusion of their work was that the eventual, periodic vortex pattern observed on the disk is the result of the merging of the fan-shaped disturbance patterns that originate from each isolated site. This was also the case in the theoretical calculations of Mack [14], which helped in further understanding this phenomenon. In continuation of the work by Wilkinson and Malik [13], Waitz and Wilkinson [15] examined the influence of unsteady external disturbances on the transition process in the rotating-disk boundary layer. These researchers found "no observable flow response to linear, localized or global acoustic forcing, either with or without roughness." A similar observation has also been made recently by Radeztsky et al [5] on the basis of their experiments on a swept-wing boundary layer. These findings suggest that the roughness-induced generation of unsteady crossflow vortices is significantly weaker than the generation of stationary vortices through the same mechanism.

The theoretical foundations for the understanding of the receptivity process, in general, were laid through the works of Goldstein [16], [17], Ruban [18], and Zavol'skii, Reutov, and Ryboushkina [19]. The basic theme in their work was an explanation of the prospective physical mechanisms, which enable the long-wavelength free-stream disturbances to excite the (typically) short-wavelength instability modes. The main catalyst in this wavelength-reduction process was identified to be the occurrence in the mean flow of components with commensurately shorter length scales. Specifically, Goldstein [16] showed how a rapidly developing mean boundary layer near the leading edge of a flat plate becomes receptive to free-stream sound, leading thereby to the formation of two-dimensional Tollmien-Schlichting waves in the region downstream. However, he also indicated how the overall effectiveness of this particular mechanism could be strongly reduced by the decay of the generated eigenmode, from the leading-edge region, up to the lower branch of the neutral stability curve. On the other hand, roughness elements of a suitable length scale can lead to receptivity directly in the vicinity of the lower branch, and thereby constitute a potentially stronger source of receptivity. The acoustic generation of Tollmien-Schlichting modes in a two-dimensional boundary layer caused by the presence of localized, but small-amplitude, variations in the surface geometry, was explained by Goldstein [17] and Ruban [18] using large Reynolds number asymptotic methods. On the other hand, Zavol'skii et al [19] used a nonasymptotic framework, based on the Orr-Sommerfeld (OS) equation, to study the receptivity due 
to distributed waviness on a flat-plate airfoil. Reviews of the work by Goldstein and Ruban, as well as the substantial subsequent work based on the asymptotic (triple-deck) theory have been given by Goldstein and Hultgren [20], Kerschen [21], and Kozlov and Ryzhov [22].

Choudhari and Streett [23] showed that, by recasting the ideas of Goldstein [17] and Ruban [18] in terms of the more familiar quasi-parallel disturbance equations, one could predict the receptivity caused by small-amplitude surface nonuniformities in a large variety of flows such as high-speed and threedimensional boundary layers. A general approach for this purpose was described in Ref. [23], and results were presented for both roughness- and suction-induced receptivity in two- and three-dimensional boundary layers. One of the applications considered was the generation of crossflow vortices; these investigators argued in the favor of a preferential excitement of stationary vortices relative to the nonstationary ones via the imperfections on the surface of the airfoil. Admittedly, their argument is only valid for shallow roughness elements, which produce only a weak perturbation to the incoming boundary layer. Nevertheless, it provides a clue to understanding the observations of Waitz and Wilkinson [15] and Radeztsky et al [5], and hence, will be summarized in the following paragraph. The problem of roughness-induced stationary disturbances in a three-dimensional boundary layer was also considered by Manuilovich [24], in the context of boundary-layer flow close to the attachment line. He found that the initial amplitude of the stationary eigenmode is many orders of magnitude smaller than the amplitude of the near-field motion directly above the localized roughness. Hence, he speculated that the transition in a swept wing boundary layer is more likely to occur because of a bypass mechanism, rather than because of the linear and nonlinear growth of crossflow instability. While this may indeed be true for roughness elements of sufficiently large height, experimental evidence suggests that the boundary layer over a typical mildly-rough surface becomes turbulent through the usual sequence of primary and secondary instabilities.

As shown in Refs. [23] and [24], the stationary vortices are generated by a direct scattering of the mean boundary-layer motion by the surface imperfections. Therefore, their initial amplitudes scale on the height of the roughness element relative to the boundary-layer thickness. On the other hand, the generation of nonstationary vortices requires an interaction between the surface roughness and the unsteady free-stream disturbances, much in the same manner as the generation of TollmienSchlichting instabilities in two-dimensional boundary layers; the latter is the topic of the (by now almost classical) theoretical works of Goldstein [17], Ruban [18], and Zavol'skii et al [19], as mentioned already. 
Accordingly, the initial amplitudes of the unsteady vortices scale on the product of the height of the roughness element and the amplitude of the unsteady free-stream disturbance. The magnitude of the free-stream fluctuations is usually quite small in a flight environment (of the order of 0.1 percent or less), which suggests that the initial amplitudes of the unsteady crossflow vortices excited via the surface roughness are likely to be significantly smaller than the amplitudes of similarly excited stationary vortices. This conjecture also appears to be supported by the earlier observations of Müller and Bippes [25] and Saric [26], who found the stationary vortices to be dominant in low turbulence wind tunnels.

However, a scaling argument alone is not sufficient to quantify the difference between the actual amplitudes of the two types of vortices in a given physical situation. To determine these amplitudes, one must also calculate the numerical coefficient that multiplies the respective scaling factor in each case. In general, these numerical coefficients will scale differently on the flow Reynolds number for the stationary and the nonstationary vortices. The above argument implicitly assumes that in the range of Reynolds numbers typically encountered in practice, this difference in Reynolds number dependence is not strong enough to fully compensate for the weakness of the unsteady forcing. Having noted this, Choudhari and Streett [23] suggested that a detailed investigation be carried out to determine (i) if and when the numerical coefficient in the unsteady case becomes relatively large, and (ii) to what extent it can offset the smallness of the unsteady vortex amplitude.

This paper attempts to fill the above deficiency by computing the initial amplitudes of both types of vortices in a few controlled situations. Within the limitations of the theoretical approach adopted herein, the author hopes that the results obtained will shed some light on the role of receptivity in determining what type of vortices will possibly dominate the primary instability stage during the transition process over a swept wing. The major limitations of this work include the consideration of only acoustic type free-stream disturbances, and the restriction to roughness distributions that correspond to sufficiently small perturbations in surface height. Another crucial assumption implicit in this work is that the crossflow modes under consideration are adequately described by the quasiparallel stability theory. While this assumption is likely to be true in a majority of cases relevant to laminar flow technology, situations can occur where crossflow modes are known to be nonparallel in certain regions of parameter space. In particular, Balakumar, Hall, and Malik [27] have shown that the unsteady eigenmodes of a rotating disk boundary layer can be nonparallel in certain frequency regimes. In addition to presenting a rather elegant theory to elucidate the structure of these nonparallel 
modes, they have also described how these nonparallel modes can be excited by the roughness on the disk surface through an interaction with unsteady external disturbances. Finally, one must note that, in general, crossflow vortices could also be excited via other receptivity mechanisms, a number of which have been identified previously in the context of a flat-plate boundary layer. These include the receptivity due to other types of surface nonuniformities, such as variations in surface suction, and/or surface admittance (Kerschen and Choudhari [28]), as well as other types of external disturbances,' such as the vorticity perturbations which are convected along with the free stream (Kerschen [29]). In addition, there might also be a mechanism for the generation of crossflow vortices, which is analogous to the leading-edge problem considered in the seminal work of Goldstein [16]. Such mechanisms are especially worthy of consideration in the present context, because the crossflow vortices become unstable relatively close to the leading edge; hence, the initial decay of the vortex modes excited near the leading edge is probably quite small.

Briefly, then, the plan of this paper is as follows. In Section 2, we describe the theoretical approach, used herein to compute the receptivity induced by a small-amplitude roughness distribution on an otherwise smooth, infinite-span swept airfoil. The basic building block for this theory is the receptivity due to a roughness distribution that is localized in one direction, but periodic along the other. In view of the infinite span of the airfoil, it appears reasonable to first assume that the periodic direction is along the airfoil span, although other types of geometries will also be considered subsequently. The receptivity caused by such "localized periodic" types of roughness distributions can be readily analyzed by extending the theory of Goldstein [17] and Ruban [18] to three-dimensional boundary layers, as discussed previously by Choudhari and Streett [23]. Thus, we will follow the nonasymptotic framework, which was originally used by Zavol'skii et al [19] for studying the distributed generation of Tollmien-Schlichting waves in a two-dimensional boundary layer over a slightly wavy surface. Similar implementations in the context of localized inhomogeneities in two-dimensional boundary layers have recently been carried out by Choudhari and Streett [30], Crouch [31], and also Fedorov, Tumin, and Zhigulev in the former U.S.S.R.; the reader may consult these latter references for additional details of the nonasymptotic adaptation. For this reason, we present a minimum of details in regard to the analysis and emphasize only the major features of the theory, plus issues that are specific to the three dimensionality of the flow. As a simplified model of the swept-wing boundary layer, we consider the two-parameter family of Falkner-Skan-Cooke (FSC) (Cooke [32]) boundary layers, which has previously 
been used in a number of theoretical and computational studies related to the linear and nonlinear dynamics of crossflow-vortex instabilities (see the works of Meyer and Kleiser [6], Mack [33], Bieler and Dallman [34], and Fischer and Dallman [35]). The results obtained from a numerically based parametric study, for a few specific profiles from the FSC family of boundary layers, are described in Section 3. Conclusions based on these results are given in Section 4.

\section{Summary of the Nonasymptotic Approach for Three-Dimensional Boundary Layers}

\subsection{Problem Definition}

As mentioned in the introduction, we shall study the receptivity of a boundary layer over a swept wing of infinite span in the context of the yawed wedge (i.e., the FSC family of flows [32]). The use of FSC profiles in modeling the stability of nonsimilar boundary layers over swept wings has been discussed in Refs. [6] and [34]. As discussed in Ref. [33], this particular choice allows for a systematic variation in both the local chordwise pressure gradient parameter (the Hartree parameter) $\beta_{H}$ and the ratio of the inviscid slip velocities in the spanwise $z^{*}$ and chordwise $x^{*}$ directions. The latter is specified in terms of the local sweep angle $\theta_{s w}$ at the location of the roughness distribution. Note that the wedge angle along the chordwise direction is related to the Hartree parameter $\beta_{H}$, and equals $(\pi / 2) \beta_{H}$ in radians. A schematic of the problem geometry is given in Fig. 1.

After the introduction of a reference length scale $\ell^{*}$, which is later identified with the chordwise position of the localized roughness distribution, and a velocity scale $\mathcal{V}^{*}$, which corresponds to the magnitude of the inviscid slip velocity at $x^{*}=\ell^{*}$, the distributions of the (nondimensional) inviscid slip velocities along the chordwise and spanwise directions can be written in the form

$$
U_{e}(x)=\cos \theta_{s w} x^{\beta_{H} /\left(2-\beta_{H}\right)}
$$

and

$$
W_{e}(x)=\text { Constant }=\sin \theta_{s w}
$$

respectively, where $x$ denotes the nondimensional chordwise coordinate scaled with respect to $\ell^{*}$. Throughout this paper, symbols with an asterisk denote dimensional quantities, and those without an asterisk denote the nondimensional ones. The subscript $e$ is used to denote the local free-stream conditions at a given chordwise location. 
Because of the infinite span of the wedge, the boundary-layer flow along the chordwise and surfacenormal directions is decoupled from the flow along the spanwise direction and is given in terms of the Falkner-Skan solutions as

$$
U_{0}=U_{e}(x) f^{\prime}(\eta)
$$

and

$$
V_{0}=-R^{-1}\left(2-\beta_{H}\right)^{-1 / 2}\left[f(\eta)-\left(1-\beta_{H}\right) \eta f^{\prime}(\eta)\right] x^{-\frac{1-\rho_{H}}{2-\beta_{H}}}
$$

respectively. Here, the similarity variable $\eta$ is defined as

$$
\eta=y^{*}\left[U_{e}^{*} /\left(2-\beta_{H}\right) \nu^{*} x^{*}\right]^{1 / 2}
$$

where $y^{*}$ is the coordinate normal to the wedge surface; $U_{e}^{*}$, the inviscid slip velocity along the chordwise direction; and $\nu^{*}$, the kinematic viscosity of the fluid. The Reynolds number $R$ is defined as

$$
R=\left(\mathcal{V}^{*} \ell^{*} / \nu^{*}\right)^{1 / 2} \quad(R>>1)
$$

and is related to the Reynolds number $R_{c}$ (based on the chordwise slip velocity at $x=1$ ) via

$$
\boldsymbol{R}=\boldsymbol{R}_{c} / \cos \theta_{\rho w}
$$

The function $f(\eta)$ in Eqs. (2.2a) and (2.2b) satisfies the boundary value problem

$$
f^{\prime \prime \prime}+f f^{\prime \prime}+\beta_{H}\left(1-f^{\prime 2}\right)=0, \quad\left(f(0)=f^{\prime}(0)=0, f^{\prime}(\infty)=1\right)
$$

The boundary-layer flow along the spanwise direction is given by

$$
W_{0}=\sin \theta_{a w} g(\eta)
$$

where $g(\eta)$ satisfies

$$
g^{\prime \prime}+f g^{\prime}=0, \quad(g(0)=0, g(\infty)=1) .
$$

As discussed in the introduction, we first consider the receptivity induced by a distribution of roughness elements that is localized in the chordwise direction at a distance $\ell^{*}$ from the leading edge. The maximum of the surface-height perturbation associated with this roughness distribution is assumed to be sufficiently small, so that the mean flow in its vicinity can be treated as a small perturbation to the incoming boundary-layer flow given by Eqs. (2.2) and (2.3) above. To facilitate the generation of the vortex instabilities in question, both the chordwise and spanwise length scales of the roughness 
distribution are assumed to be much shorter than $\ell^{*}$, specifically, in the same range as the corresponding wavelengths of the crossflow modes at $x^{*}=\ell^{*}$. The small-height approximation implies that the different Fourier components of the disturbance motion along the spanwise direction are decoupled from each other to the required level of accuracy. Hence, without any loss of generality, one may assume that the wall-height distribution (relative to the underlying flat surface) is periodic along the spanwise direction and is given by

$$
h_{w}^{*}\left(x^{*}, z^{*}\right) / L^{*}=\epsilon_{w} h_{w}(X) e^{i \beta_{w} Z} ; \quad \epsilon_{w}<<1
$$

In Eq. (2.4a), we have introduced an additional length scale

$$
L^{*}=\ell^{*} / R_{c}
$$

which is proportional to the thickness of the unperturbed chordwise boundary layer at the location of the roughness distribution. This shorter length scale is used to scale both the surface height itself and its variation along the streamwise and spanwise directions. Thus, the latter is specified in terms of the local streamwise coordinate $X=\left(x^{*}-\ell^{*}\right) / L^{*}$ (instead of the global variable $x$ in Eqs. (2.2) and (2.3)), and the "fast" spanwise coordinate $Z=z^{*} / L^{*}$. The small parameter $\epsilon_{w}$ in Eq. (2.4a) characterizes the magnitude of the wall-height perturbation, and $\beta_{w} \equiv \beta_{w}^{*} L^{*}$ represents the nondimensional wavenumber that corresponds to the spanwise-periodic distribution. The function $h_{w}(X)$ specifies the streamwise shape of the roughness distribution in terms of the local coordinate $X$.

The unsteady perturbation upstream of the wedge is assumed to be a small-amplitude acoustic disturbance with a frequency of $\omega^{*}$ and a perturbation-velocity vector that lies in the same plane as the incoming steady flow and the leading edge of the wedge. As we shall subsequently observe, this latter assumption is not necessary for this analysis; however, it does simplify the ensuing presentation. Because the acoustic wavelength is infinite in the low Mach number limit, the outer unsteady motion is quasi-steady in nature; therefore, the associated unsteady slip-velocity distribution can be expressed in the form

$$
u_{a c, e}^{*}\left(x^{*}\right) / \mathcal{V}_{a c}^{*}=\cos \theta_{a c} x^{\beta_{H} /\left(2-\beta_{H}\right)} e^{-i \omega t}
$$

and

$$
w_{a c, e}^{*}\left(x^{*}\right) / \mathcal{V}_{a c}^{*}=\text { Constant }=\sin \theta_{a c} e^{-i \omega t}
$$

where $\mathcal{V}_{a c}^{*}$ denotes the magnitude of the unsteady slip velocity at the roughness location $(x=1)$, such 
that $\epsilon_{a c} \equiv \mathcal{V}_{a c}^{*} / \mathcal{V}^{*}<<1$. The nondimensional frequency $\omega$ and time $t$ have been scaled by $\mathcal{V}^{*} / L^{*}$ and its inverse respectively.

This completes the definition of the problem under consideration. The objective of the receptivity analysis now is to solve for the disturbance motion in the vicinity of the roughness distribution, so that it becomes possible to estimate the initial amplitudes of both the steady crossflow vortex (generated directly by the surface nonuniformity) and the unsteady mode of instability (generated via the interaction of the nonuniformity with the unsteady disturbance in the free stream). One may reiterate that the physical mechanisms behind both these receptivity problems are the same, in a general sense at least, as those pointed out by Goldstein [17], Ruban [18], and Zavol'skii et al [19], in that the roughness distribution provides the appropriate length scales necessary for the generation of each instability mode in question. Hence, the stationary vortex mode is excited by a direct "scattering" of the mean boundary-layer flow at the roughness location, while the unsteady vortex is generated by a similar scattering of the unsteady motion associated with the free-stream disturbance.

\subsection{Perturbation Scheme, and Extraction of the Steady and Unsteady Eigenmodes for a Spanwise-Periodic Roughness Distribution}

\subsubsection{Perturbation Scheme}

As shown by Goldstein [17], Ruban [18], and Zavol'skii et al [19], the analysis of roughnessinduced receptivity simplifies considerably after the small-disturbance approximation is invoked $\left(\epsilon_{w}<<\right.$ $\left.1, \epsilon_{a c}<<1\right)$ and the disparity is exploited between the length scale $L^{*}$ of the roughness distribution and the streamwise length scale $\ell^{*}$ of the unperturbed mean boundary layer. Specifically, if $\mathcal{V}^{*}$ and $\rho^{*} \mathcal{V}^{* 2}$ are used to nondimensionalize the total velocities and pressure, respectively, in the vicinity of the roughness distribution, then we can expand the resulting array of dependent variables $\mathbf{Q} \equiv(U, V, W, P)$ in the form

$$
\begin{aligned}
\mathbf{Q}(X, Y, Z, t) & =\mathbf{Q}_{0}(Y)+\epsilon_{w} \mathbf{Q}_{w}(X, Y) e^{i \beta_{w} Z}+\epsilon_{a c} \mathbf{Q}_{a c}(Y) e^{-i \omega t} \\
& +\epsilon_{w} \epsilon_{a c} \mathbf{Q}_{w, a c}(X, Y) e^{i\left(\beta_{w} Z-\omega t\right)}+O\left(\epsilon_{w}^{2}, \epsilon_{a c}^{2}, R^{-1}\right)
\end{aligned}
$$

where $Y$ denotes the wedge-normal coordinate scaled with respect to $L^{*}$. The suffixes $w$ and ac indicate the first-order perturbations caused by the wall roughness and the free-stream acoustic wave, respectively; the combined suffix $w$, ac represents the mutual interaction between the two first-order perturbations. In writing this expansion, we have already utilized the local nature of the region of 
receptivity along the chordwise direction $\left(L^{*} / \ell^{*}<<1\right)$ by replacing the unperturbed boundary-layer flow $\left(U_{0}(x, Y), V_{0}(x, Y), W_{0}(x, Y), P_{0}(x)\right)$ by its local quasi-parallel approximation $\mathbf{Q}_{0}(Y)=\left(U_{0}(x=\right.$ $\left.1, Y), 0, W_{0}(x=1, Y), P_{0}(x=1)\right)$, which is accurate to within an error of $O\left(R^{-1}\right)$. Also implicit in the stated form of perturbations at $O\left(\epsilon_{w}\right)$ and $O\left(\epsilon_{w} \epsilon_{a c}\right)$ is the assumption that the unperturbed profile $\mathbf{Q}_{0}(Y)$ is not absolutely unstable. The calculation of these perturbations, and the extraction of the generated instability modes from them is described in the following two subsections.

\subsubsection{Extraction of the stationary crossflow mode}

The mean-flow disturbance caused by the wall nonuniformity, $\mathbf{Q}_{w}$, satisfies the steady Navier-Stokes equations, which are linearized about the parallel mean flow, $\mathbf{Q}_{0}(Y)$. The boundary conditions that accompany this homogeneous set of equations include the usual constraints of zero normal velocity and no slip at the roughness boundary $Y=\epsilon_{w} h_{w}(X) e^{i \beta_{w} Z}$ and the zero-disturbance condition as $Y \rightarrow \infty$. Specification of the appropriate boundary conditions along the chordwise $(X)$ direction requires causality considerations, as discussed further below. These considerations ensure that the computed solution for the mean-flow perturbation is physically realizable as the large-time limit of the solution to a corresponding time-dependent problem.

Within the linear quasi-parallel approximation, the perturbation $V_{w}$ in the vertical velocity is decoupled from the perturbation $\Omega_{Y_{w}} \equiv \partial U_{w} / \partial Z-\partial W_{w} / \partial X$ in the vertical vorticity. This decoupling enables one to obtain a single equation for $V_{w}$, which is fourth order in $Y$ and, furthermore, reduces to the time-independent form of the Orr-Sommerfeld (OS) equation in the Fourier transform $(X \rightarrow \alpha)$ space. Thus, we have

$$
\begin{gathered}
\mathcal{L}_{O S}^{\left(\alpha, \beta_{w}, 0, R\right)} \bar{V}_{w}=0 \\
\left(\mathcal{C}_{O S}^{(\alpha, \beta, \omega, R)}=i\left(\alpha U_{0}+\beta W_{0}-\omega\right)\left[D^{2}-\left(\alpha^{2}+\beta^{2}\right)\right]-i\left(\alpha U_{0}^{\prime \prime}+\beta W_{0}^{\prime \prime}\right)-\frac{1}{R}\left[D^{2}-\left(\alpha^{2}+\beta^{2}\right)\right]^{2}\right)
\end{gathered}
$$

wherein we have used an overbar to denote the Fourier transformed perturbation

$$
\bar{V}_{w}(\alpha, Y)=\frac{1}{\sqrt{2 \pi}} \int_{-\infty}^{\infty} e^{-i \alpha X} V_{w}(X, Y) d X
$$

the operator $D$, as well as the primes, denotes differentiation with respect to the $Y$ coordinate. Linearization (and a transfer to $Y=0$ ) of the surface boundary conditions leads to the inhomogeneous set of conditions

$$
\bar{V}_{w}=0, \quad \bar{V}_{w}^{\prime}=i\left(\alpha U_{0}^{\prime}(0)+\beta_{w} W_{0}^{\prime}(0)\right) \bar{h}_{w}(\alpha) \text { at } Y=0
$$


Finally, of course, we have the zero-disturbance conditions far away from the wall, viz.,

$$
\bar{V}_{w}, \bar{V}_{w}^{\prime} \rightarrow 0 \text { as } Y \rightarrow \infty
$$

After the vertical velocity perturbation has been determined by solving Eqs. (2.7a),(2.7c), and (2.7d), the combination $\left(\alpha \vec{U}_{w}+\beta_{w} \bar{W}_{w}\right)$ of the horizontal velocity components follows trivially from the continuity equation

$$
i\left(\alpha \bar{U}_{w}+\beta_{w} \bar{W}_{w}\right)+\bar{V}_{w}^{\prime}=0
$$

The individual velocity transforms $\bar{U}_{w}$ and $\bar{W}_{w}$ can also be determined by further solving the vertical vorticity equation (i.e., the Squire equation [36]) in the Fourier transform space, namely,

$$
\begin{gathered}
\mathcal{L}_{\text {Squire }}^{(\alpha, \beta, 0, R)}\left(\beta_{w} \bar{U}_{w}-\alpha \bar{W}_{w}\right)=-\left(\beta_{w} U_{0}^{\prime}-\alpha W_{0}^{\prime}\right) \bar{V}_{w} \\
\left(\mathcal{L}_{\text {Squire }}^{(\alpha, \beta, w, R)}=i\left(\alpha U_{0}+\beta W_{0}-\omega\right)-\frac{1}{R}\left[D^{2}-\left(\alpha^{2}+\beta^{2}\right)\right]\right)
\end{gathered}
$$

with the inhomogeneous boundary condition

$$
\beta_{w} \bar{U}_{w}-\alpha \bar{W}_{w}=-\left(\beta_{w} U_{0}^{\prime}(0)-\alpha W_{0}^{\prime}(0)\right) \text { at } Y=0
$$

plus the homogeneous condition

$$
\beta_{w} \bar{U}_{w}-\alpha \bar{W}_{w} \rightarrow 0 \text { as } Y \rightarrow \infty
$$

Finally, the pressure perturbation $\bar{P}_{w}$ can be calculated from either the linearized $X$-momentum equation or by solving the linearized form of the usual Poisson equation that governs the pressure distribution in an incompressible flow. However, if one is not interested in the total mean-flow perturbation produced by the surface roughness, but only in the crossflow instability modes generated as a part thereof, then it is sufficient to just solve for the perturbation in any single flow variable, and this, in our case, will be $V_{w}$. Having identified the amplitude of the vertical velocity perturbation corresponding to the crossflow mode of interest from the solution for $V_{w}$, it is easy to determine the corresponding perturbations in other flow variables by using the eigenfunction properties for this crossflow mode. (Refer to the discussion related to Eq. (2.10) below.)

One may observe that the Fourier transform solution $\bar{V}_{w}(\alpha, Y)$ can be computed from $(2.7 \mathrm{a}),(2.7 \mathrm{c})$, and (2.7d) without having to account for causality in the problem. However, the latter considerations arise during the inversion of the Fourier transform,

$$
V_{w}(X, Y)=\frac{1}{\sqrt{2 \pi}} \int_{\Gamma} e^{i \alpha X} \bar{V}_{w}(Y) d \alpha,
$$


via the specification of the inversion contour $\Gamma$ in the complex $\alpha$ plane. Basically, the value of the integral in (2.9) is determined by contributions from the poles and branch cuts of the integrand, $\bar{V}_{w}$, which include the singularities of the forcing function $\bar{h}_{w}(\alpha)$ in $2.7 \mathrm{a}, \mathrm{c}$, plus the discrete and continuum eigenmodes of the homogeneous, steady OS boundary value problem. In particular, one of the discrete OS modes, denoted here by $\alpha_{i n s}\left(\beta_{w}, \omega=0 ; R\right)$, represents the potentially unstable stationary crossflow mode, which was identified in the stability calculations of Mack [33], and Dallman and Bieler [34]. However, whether the contribution due to this mode is relevant to the upstream or the downstream side of the roughness distribution will be determined by whether or not the causal position of $\Gamma$ lies above the first-order pole singularity at $\alpha=\alpha_{i n s}\left(\beta_{w}, 0 ; R\right)$. This usually requires a rigorous scrutiny along the lines of the Briggs-Bers criterion [37] [38], which, as originally derived, is only applicable to twodimensional disturbances in a two-dimensional flow. Extension of this criterion to a three-dimensional boundary layer has not been attempted yet, even though the problem simplifies considerably in our present case, on account of the restriction to a spanwise periodic roughness distribution. Hence, in our calculations, we will simply assume that the crossflow instability is convective in nature, which guarantees that the generated crossflow vortex always appears on the downstream side of its source. Cases where this may not be true are always possible, but, certainly, there is a considerable experimental evidence thus far which tends to support the validity of this assumption - see Huerre and Monkowitz, [39].

The residue contribution to the integral in (2.9), from the pole singularity of the integrand at $\alpha=\alpha_{\text {ins }}\left(\beta_{w}, \omega=0 ; R\right)$, can be ascertained through a numerical evaluation of the Fourier transform solution, $\bar{V}_{w}(\alpha, Y)$, in the vicinity of this wavenumber location (see the early work by Gaster [40], and Tam [41] in this context). The corresponding part of the mean-flow perturbation in an arbitrary flow variable $q$ can be expressed in the same form as Goldstein [17], namely,

$$
q_{s}=\bar{h}_{w}\left(\alpha_{i n s}\left(\beta_{w}, 0, R\right)\right) \Lambda_{s}\left(\beta_{w}, R\right) E_{q}\left(Y ; \beta_{w}, 0, R\right) e^{\left.i\left[\alpha_{i n s}\left(\beta_{w}, 0, R\right) X+\beta_{w} Z\right)\right]}
$$

where the subscript $s$ denotes a stationary vortex, and $\bar{h}_{w}\left(\alpha_{i n s}\left(\beta_{w}, 0, R\right)\right)$ is the amplitude of the Fourier component of the (scaled) roughness geometry which is locally resonant with the stationary vortex mode. The factor $\Lambda_{s}\left(\beta_{w}, R\right)$ is, effectively, a Green's function for the roughness-induced stationary receptivity problem. It is an intrinsic property of the local boundary-layer profile, characterizing the efficiency of the local receptivity process, much in the same manner as the eigenvalue $\alpha_{i n s}\left(\beta_{w}, 0, R\right)$ characterizes the stability of this profile. Hence, one may use this "efficiency" function to gauge the 
"receptivity properties" of the boundary layer at different spanwise wavenumbers, and/or chordwise locations, irrespective of the geometry of the roughness distribution. The function $E_{q}\left(Y, \beta_{w}, 0, R\right)$ denotes the stationary-vortex eigenfunction, which we assume to have been normalized in such a way that the maximum magnitude of $E_{\cos \theta_{o w}} U+\sin \theta_{s w} W$, i.e., the eigenfunction associated with the velocity perturbation along the local inviscid streamline, is equal to unity. This normalization implies that the effective initial amplitude of the stationary vortex, based on this latter quantity, is given by

$$
\left(\cos \theta_{s w} u_{s}^{*}+\sin \theta_{s w} w_{s}^{*}\right) / \mathcal{V}^{*}=\epsilon_{w} \bar{h}_{w}\left(\alpha_{i n s}\left(\beta_{w}, 0, R\right)\right) \Lambda_{s}\left(\beta_{w}, R\right)
$$

\subsubsection{Extraction of the unsteady - crossflow and Squire - eigenmodes}

We now consider the generation of an unsteady crossflow vortex via the local scattering of $\mathbf{Q}_{a c}$, i.e., the basic unsteady motion, corresponding to the signature of the acoustic disturbance within the unperturbed, mean boundary layer. If we ignore the relatively narrow band of small-frequency crossflow vortices with $\omega=O\left(R^{-1}\right)$ or less, then to the leading order of approximation in the Reynolds number $R$, the acoustic-signature field $\mathbf{Q}_{a c}(Y)$ is given by the quasi-parallel, Stokes shear-wave solution,

$$
\begin{gathered}
U_{a c}=\cos \theta_{a c}\left(1-\exp \left[i s_{\omega}(i \omega R)^{1 / 2} Y\right]\right) x^{\beta_{H} /\left(2-\beta_{H}\right)} e^{-i \omega t} \\
V_{a c}=0 \\
W_{a c}=\sin \theta_{a c}\left(1-\exp \left[i s_{\omega}(i \omega R)^{1 / 2} Y\right]\right) e^{-i \omega t} \\
P_{a c}=i \omega\left(\cos \theta_{a c} X+\sin \theta_{a c} Z\right) e^{-i \omega t}
\end{gathered}
$$

where $s_{\omega}= \pm 1$, depending on whether the frequency parameter $\omega$ is positive or negative.

We had previously alluded to the fact that the localized receptivity of a low-speed boundary layer to an acoustic wave of sufficiently high frequency is independent of the upstream form of the free-stream disturbance. The rationale behind this observation may be gleamed from the form of the Stokes-wave solution above, which is completely determined by the local unsteady slip velocity, being independent of both the previous history of the free-stream disturbance and the local boundary-layer motion. For frequencies that correspond to $\omega=O\left(R^{-1}\right)$ or less, the Stokes-wave approximation becomes invalid. However, the other aspects of this receptivity analysis remain exactly the same. Hence, if one needs to compute the receptivity characteristics in this range of frequencies, it is sufficient to replace the 
solution in (2.11a-d) with the correct solution for the local acoustic signature, and then follow through the remaining parts of the receptivity analysis similar to that described underneath for the largefrequency case. As one can infer from the work of Lam and Rott [42], the correct asymptotic form of the acoustic signature field at lower frequencies can be obtained by solving the linearized, threedimensional, unsteady boundary-layer equations. It is obvious that due to the parabolic nature of these equations, the receptivity calculation in this range of frequencies depends upon both the upstream form of the free-stream disturbance as well as the mean boundary-layer motion.

The generation of an unsteady vortex instability may now be viewed as the "tuning" of the locally uniform acoustic-signature field to the wavenumber vector, $\alpha_{i n s}\left(\beta_{w}, \omega ; R\right)$, of the unsteady vortex mode, via the corresponding Fourier component, $\bar{h}_{w}\left(\alpha_{i n s}\left(\beta_{w}, \omega ; R\right)\right)$, of the surface roughness distribution. As pointed out by Zavol'skii et al [19] and Goldstein [17], this roughness-induced tuning involves both (i) a direct scattering of the Stokes wave at the rough surface, and (ii) an indirect scattering via the mean-flow perturbation $\mathbf{Q}_{w}$. This mean-flow perturbation includes the stationary crossflow mode, as discussed above; but, the presence of this mode does not influence the excitation of the unsteady mode, since the two wavenumbers, $\alpha_{i n s}\left(\beta_{w}, \omega=0 ; R\right)$ and $\alpha_{i n s}\left(\beta_{w}, \omega ; R\right)$, are not equal, in general. Thus, any unsteady receptivity through the indirect scattering mechanism is entirely due to the remaining part of the mean-flow perturbation, which remains localized in the vicinity of the roughness distribution. Of course, if and when the steady and unsteady vortices have the same streamwise wavenumber, we have a triad resonance between the two crossflow vortices and the acoustic-signature field, and this can lead to a sustained generation of the unsteady mode even in the smooth region downstream of the roughness distribution. This is an exceptional case, however, and will not be considered here.

Along the lines of the mean-flow perturbation $\mathbf{Q}_{w}$, it can be shown that the Fourier transform of the vertical velocity perturbation associated with the unsteady scattered field $\mathbf{Q}_{w, a c}$ is governed by the inhomogeneous OS problem,

$$
\begin{gathered}
\mathcal{L}_{O S}^{\alpha, \beta_{w}, \omega, R} \bar{V}_{w, a c}=-i\left(\cos \theta_{a c} \alpha+\sin \theta_{a c} \beta_{w}\right)\left\{\left(1-\exp \left[i s_{\omega}(i \omega R)^{1 / 2} Y\right]\right)\left[D^{2}-\left(\alpha^{2}+\beta_{w}^{2}\right)\right]\right. \\
\left.-i \omega R \exp \left[i s_{\omega}(i \omega R)^{1 / 2} Y\right]\right\} \vec{V}_{w} \\
\bar{V}_{w, a c}=0, \bar{V}_{w, a c}^{\prime}=s_{\omega}(i \omega R)^{1 / 2}\left(\cos \theta_{a c} \alpha+\sin \theta_{a c} \beta_{w}\right) \text { at } Y=0 \\
\bar{V}_{w, a c}, \bar{V}_{w, a c}^{\prime} \rightarrow 0 \text { as } Y \rightarrow \infty
\end{gathered}
$$


Similarly, the Fourier transform of the unsteady vertical-vorticity field satisfies the inhomogeneous Squire equation,

$$
\begin{gathered}
\mathcal{L}_{S q u i r e}^{\alpha, \beta_{w}, \omega, R}\left(\beta_{w} U_{w, a c}-\alpha W_{w, a c}\right)=-\left\{\left(\beta_{w} U_{0}^{\prime}-\alpha W_{0}^{\prime}\right) \bar{V}_{w, a c}\right. \\
+i\left(\cos \theta_{a c} \alpha+\sin \theta_{a c} \beta_{w}\right)\left(1-\exp \left[i s_{\omega}(i \omega R)^{1 / 2} Y\right]\right)\left(\beta_{w} \bar{U}_{w}-\alpha \bar{W}_{w}\right) \\
\left.-i\left(\cos \theta_{a c} \beta_{w}-\sin \theta_{a c} \alpha\right) s_{\omega}(i \omega R)^{1 / 2} \exp \left[i s_{\omega}(i \omega R)^{1 / 2} Y\right] \bar{V}_{w}\right\},
\end{gathered}
$$

along with the inhomogeneous boundary condition,

$$
\left(\beta_{w} \bar{U}_{w, a c}-\alpha \bar{W}_{w, a c}\right)=i s_{w}(i \omega R)^{1 / 2}\left(\cos \theta_{a c} \beta_{w}-\sin \theta_{a c} \alpha\right) \text { at } Y=0
$$

plus the homogeneous condition,

$$
\left(\beta_{w} \bar{U}_{w, a c}-\alpha \bar{W}_{w, a c}\right) \rightarrow 0 \text { as } Y \rightarrow \infty
$$

Again, the perturbations associated with the generated unsteady vortex mode can be separated out as the residue contribution corresponding to the pole at the relevant unsteady eigenvalue, $\alpha=$ $\alpha_{\text {ins }}\left(\beta_{w}, \omega, R\right)$. This yields

$$
q_{u}=\bar{h}_{w}\left(\alpha_{i n s}\left(\beta_{w}, \omega, R\right)\right) \Lambda_{u}\left(\beta_{w}, \omega, R, \theta_{a c}\right) E_{q}\left(Y, \beta_{w}, \omega, R\right) e^{i\left[\alpha_{i n s}\left(\beta_{w}, \omega, R\right) X+\beta_{w} Z-\omega t\right]},
$$

where the subscript $u$ denotes an unsteady mode, and the interpretation of the geometry function $\bar{h}_{w}$, and the efficiency function $\Lambda_{u}$, is quite similar to the interpretation of the respective functions in the stationary case examined previously, except that in addition to its dependence on the mean boundary-layer properties (analogous to $\Lambda_{s}$ ) and the frequency parameter $\omega$, the efficiency function $\Lambda_{v}$ also depends upon the acoustic-wave orientation $\theta_{a c}$, in view of the dependence of the inhomogeneous terms in the OS boundary value problem $(2.12 \mathrm{a}-\mathrm{c})$ on $\theta_{a c}$. Thus, the nondimensional initial amplitude of the nonstationary mode, based, again, on the maximum velocity perturbation along the external inviscid streamline, is given by the product

$$
\left(\cos \theta_{s w} u_{u}^{*}+\sin \theta_{s w} w_{u}^{*}\right) / \mathcal{V}_{a c}^{*}=\epsilon_{w} \vec{h}_{w}\left(\alpha_{i n s}\left(\beta_{w}, \omega, R\right)\right) \Lambda_{u}\left(\beta_{w}, \omega, R, \theta_{a c}\right)
$$

It is easy to see that the dependence of unsteady receptivity on the acoustic wave orientation is only manifested through a single multiplicative factor $\left(\cos \theta_{a c} \alpha+\sin \theta_{a c} \beta_{w}\right)$ in both the volumetric (2.12a), as well as boundary (2.12b) inhomogeneities. In view of this, it is possible to express $\Lambda_{u}\left(\beta_{w}, \omega, R, \theta_{a c}\right)$ in the form

$$
\Lambda_{u}\left(\beta_{w}, \omega, R, \theta_{a c}\right)=\frac{\alpha_{i n s}}{\cos \theta_{a c} \alpha_{i n s}+\sin \theta_{a c} \beta_{w}} \Lambda_{u}\left(\beta_{w}, \omega, R, 0\right)
$$


Equation (2.15) implies that the efficiency function is proportional to the projection of the local unsteady slip velocity along the direction of the wavenumber vector corresponding to the instability wave. The simplicity of this result stems from the isotropy (in the $X-Z$ plane) of the shape function that describes the variation in the Stokes-wave solution along the wall-normal direction. The fact that the result (2.15) is identical to an earlier result by Choudhari and Kerschen [43] for the case of a two-dimensional boundary layer should not have come as a surprise, since the Stokes-wave solution is independent of the mean boundary-layer motion, as mentioned previously.

One may observe that it is also possible to compute the amplitude of the Squire mode at a spanwise wavenumber of $\beta_{w}$, and frequency $\omega$, by solving the inhomogeneous Squire problem (2.13a-c). However, these modes are always damped [36]. Moreover, their role in the transition process is also not firmly established, although, for two-dimensional boundary layers, they might be playing the role of dormant secondary instabilities of the subharmonic type - see, especially, the insightful work of Herbert [44] in this context. Unfortunately, the role of these Squire modes in the transition of three-dimensional boundary layers is even less clear, and, therefore, we have not attempted to compute their initial amplitudes in this paper. The generation of Squire modes in a two-dimensional boundary layer, due to a three-dimensional surface nonuniformity, has been examined, however, and will be reported in a separate paper.

The reader may also note that a stability calculation for the spatial growth rate of a threedimensional instability mode requires information about the direction in which this normal mode amplifies. A typical assumption made for infinite-span geometries is that the amplification occurs purely along the chordwise direction [33]. This particular choice is supported by concepts borrowed from the conservative wave theory, in particular, the irrotationality requirement for the wavenumber vector corresponding to the instability mode. However, other choices are possible as well and, indeed, have been suggested in the literature on stability (see Nayfeh [45]). This nonuniqueness basically arises from a lack of knowledge concerning the source of this instability mode. In a receptivity calculation, there is no such ambiguity. Thus, in the present case, the spanwise periodic nature of the roughness distribution automatically implies that the generated vortex (stationary, as well as nonstationary) will amplify along the chordwise direction. 


\subsection{Application of the Theory to Other Roughness Geometries}

\subsubsection{Skewed Arrays of Compact Roughness Elements}

One common occurence for the spanwise-periodic roughness distribution considered thus far corresponds to a set of compact roughness elements placed at equal distance along the airfoil span. Such an array contains energy in all harmonics of the fundamental; the analysis above represents the. receptivity caused by one such harmonic. In practice, however, it is also possible to encounter roughness arrays which are skewed with respect to the spanwise direction (Fig. 2). Because the flow Reynolds number varies continuously along the length of a skewed array, the resulting instability motion is somewhat more complex than in the spanwise periodic case. However, at least on a local basis, it can be treated as a single crossflow vortex that decays or amplifies in a direction orthogonal to the skewed array, rather than in the chordwise direction. Of course, for a fixed spacing between each pair of adjacent roughness elements, the wavelength of the crossflow vortex generated by a skewed array is different from that generated by a spanwise-periodic one.

A crossflow mode of the same wavelength and axial orientation can be excited as a fundamental harmonic with the skewed roughness array only when this mode is locally neutral, and one tunes the interelement spacing along the array to match the wavelength of the vortex mode in that direction. To compute the initial amplitude of the vortex in this case, one could simply rotate the axes of the coordinate system so that the new axis that corresponds to the periodic direction is aligned with the roughness array and then proceed with the residue calculation along the other (i.e., nonperiodic) direction, as before. However, no additional calculation is necessary for this purpose because the initial amplitude of a given (neutral) vortex mode excited by a skewed roughness array is related to the amplitude of the same mode in the spanwise periodic case by the relation

$$
\frac{q\left(\theta_{r}\right)}{q\left(\theta_{r}=0\right)}=\frac{\cos \theta_{r} \beta_{w}-\sin \theta_{r} \alpha_{i n s}}{\beta_{w}} \frac{1}{\cos \theta_{r}-\frac{\partial \alpha_{i n 1}}{\partial \beta_{w}} \sin \theta_{r}},
$$

where the angle $\theta_{r}$ is the orientation of the skewed roughness array with respect to the spanwise direction (Fig. 2).

As seen from Eq. (2.16), the ratio $q\left(\theta_{r}\right) / q\left(\theta_{r}=0\right)$ can be expressed as a product of two other ratios, each of which can be given a simple physical interpretation. The first of these ratios corresponds to the ratio of the number of roughness elements per unit length along both the skewed and the spanwiseperiodic arrays, respectively. This ratio arises from the fact that the vortex mode under consideration 
has different wavelengths along the two arrays; hence, the amplitude of the fundamental harmonic (i.e., the geometry factor $\bar{h}_{w}$ ) has a different value in each case. The other factor on the right-hand side of Eq. (2.16) relates the residues along two different nonperiodic directions for a fixed forcing in the Fourier transform space (i.e., for the same geometry factor $\bar{h}_{w}$ ). Thus, this factor reflects the varying sensitivity of the boundary-layer flow to roughness arrays with different orientations.

Because the first of the above two ratios is proportional to the projection of the wavenumber vector $\left(\alpha_{i n s}, \beta_{w}\right)$ along the roughness array, it has its maximum magnitude when these two directions are aligned with each other. On the other hand, this factor reaches its minimum value of zero when the array is perpendicular to the wavenumber vector of the vortex, which essentially reflects the fact that the wavelength of the vortex mode along this direction tends to infinity so that any array with a finite periodicity along this direction cannot excite this mode as a fundamental harmonic. The second ratio in Eq. (2.16) is inversely proportional to the projection of the group velocity vector (which corresponds to the vortex mode of interest) on the normal to the roughness array. Therefore, its magnitude is minimum when the roughness array is orthogonal to the group velocity vector, but maximum when the roughness array is nearly aligned with the latter direction. Again, one should keep in mind that in the case of a skewed roughness array, it is more accurate to treat the total amplitude of the generated crossflow mode as the summation of the respective contributions from each isolated roughness element. Therefore, the above results may be influenced by the additional desynchronization between such individual contributions by virtue of the slow variation in the mean boundary-layer properties along the array (see, for instance, Refs. [19] and [46] for the discussion of a related problem).

\subsubsection{A Single, Isolated Roughness Element}

Now, let us discuss the application of the theory to the problem of receptivity due to an isolated three-dimensional roughness element. The analogous problem for a two-dimensional boundary layer was examined by Choudhari and Kerschen [43]. As discussed by these authors, the instability motion generated by a doubly-compact source of this type can be represented as the integral over a continuous spectrum of Fourier modes along some arbitrarily chosen physical direction. Thus, while the initial amplitude for each Fourier mode can be related to the efficiency functions $\Lambda_{s}$ and $\Lambda_{u}$ above, the efficiency function at any single wavenumber need not have any direct bearing on the total instability amplitude at any given location. Fortunately, an application of the steepest descent method [47] shows 
that, sufficiently farther downstream of the source, the instability motion along each ray that originates from the source is dominated by a narrow band of wavenumbers, which is centered on the mode which has its group-velocity vector directed along this ray. Therefore, the instability amplitude along this ray can, again, be related to the efficiency function for this dominant mode. A steepest descent calculation for a white-noise source in a rotating-disk boundary layer was given by Mack [14].

\subsubsection{Roughness Patterns That are Nonlocalized in Both Directions}

Now that we have discussed the application of the theory to roughness distributions that are compact in either one or both directions, let us briefly examine the issue of receptivity attributed to roughness distributions that are noncompact in both $X$ and $Z$. Such distributions can correspond to, for example, a two-dimensional lattice of discrete roughness elements, a known waviness in the surface geometry, or a completely random distribution of roughness height that is known only in terms of its joint probability distribution function. As discussed by Choudhari and Streett [30], the generation of instabilities in distributed regions of this type can still be analyzed by a relatively simple extension of the localized receptivity results. The idea here is to treat the receptivity as a weighted integral over the contributions from each infinitesimal subregion along the direction of instability growth. Each local contribution is related to the value of the efficiency function above, whereas the weighting function basically accounts for the phase shift and the amplification, with respect to some fixed reference station. Thus, for instance, the dimensional amplitude of the chordwise-velocity perturbation associated with an unsteady vortex mode is determined by the integral

$$
\begin{gathered}
u_{u}^{*}=\frac{1}{\sqrt{2 \pi}} e^{i\left[\Theta_{i n s}\left(x^{*}\right)+\beta_{w^{*}}^{*} z^{*}-\omega^{*} t^{*}\right]} \\
\int^{x^{*}} E_{u}\left(Y_{\max }, \beta_{w}^{*}, \omega^{*}, \tilde{R}\right) \Lambda_{u}\left(\beta_{w}^{*}, \omega^{*}, \tilde{R}, \theta_{a c}\left(\tilde{x}^{*}\right)\right) \frac{h_{w}^{*}\left(\tilde{x}^{*}\right)}{\tilde{R}_{c}^{-1} \tilde{x}^{*}} \mathcal{V}_{a c, e}^{*}\left(\tilde{x}^{*}\right) e^{-i \Theta_{i n s}\left(\tilde{x}^{*}\right)} H\left(x^{*}-\tilde{x}^{*}\right) \frac{d \tilde{x}^{*}}{\tilde{R}_{c}^{-1} \tilde{x}^{*}}
\end{gathered}
$$

where $Y_{\max }$ denotes the maximum of $\left|E_{u}\right|$ along the wall-normal direction at a given station, and

$$
\Theta_{i n s}\left(x^{*}\right) \equiv \int^{x^{*}} \alpha_{i n s}^{*}\left(\beta_{w}^{*}, \omega^{*}, \tilde{x}^{*}\right) d \tilde{x}^{*}=\left(2-\beta_{H}\right) \int^{R_{c}} \alpha_{i n s}\left(\beta_{w}^{*}, \omega^{*}, \tilde{R}_{c}\right) d \tilde{R}_{c}
$$

characterizes the chordwise variation (with respect to some fixed reference location) in the complex phase of the instability mode under consideration. Note that the presence of dimensional quantities $\beta_{w}^{*}$ and $\omega^{*}$ in the list of arguments for $E_{u}, \Lambda_{u}$, and $\alpha_{i n s}$ indicates that the physical frequency and 
spanwise wavenumber are kept fixed across the region of integration, rather than the nondimensional values $\omega$ and $\beta_{w}$, which were based on local length and velocity scales. From now on, we will take this for granted, and omit both $\beta_{w}^{*}$ and $\omega^{*}$ from the above lists for brevity of notation.

Zavol'skii et al [19], Tumin and Fedorov [48], and Choudhari [46] have shown that the value of an integral of this type is dominated by the contributions from a relatively narrow region in the vicinity of the lower branch location. The concentration of receptivity near the lower branch region was also noted by Crouch [49] in his work. As shown in Refs. [19], [46], and [48], the streamwise length scale of this narrow region is intermediate to the wavelength of the instability wave at the lower branch location, and the longer length scale over which the mean boundary layer evolves. This further simplifies the analysis by allowing one to neglect the variations in the efficiency function $\Lambda_{u}$ across this region and to approximate the instability wavenumber $\alpha_{i n s}$ by a linear Taylor-series approximation in the chordwise coordinate about the lower branch location. Closed-form expressions can now be derived for certain special types of geometries. In particular, if we assume that the roughness distribution corresponds to a sinusoidal shape of the form

$$
h_{w}^{*}\left(x^{*}, z^{*}\right) / h_{0}^{*}=e^{i\left[\alpha_{w}^{*}\left(x^{*}-x_{i, b}^{*}\right)+\beta_{w}^{*} z^{*}+\phi_{0}\right]},
$$

then the maximum of the chordwise-velocity perturbation at locations that are sufficiently farther downstream of the lower branch station is given by

$\frac{u_{u}^{*}}{\mathcal{V}_{a c}^{*}}=\frac{R_{h_{0}}}{R_{l . b .}} \sqrt{\frac{2}{i D_{\alpha}}} E_{u}\left(Y_{m a x}, R_{l . b .}\right) \Lambda_{u}\left(R_{l . b .}, \theta_{a c}\left(x_{l . b .}^{*}\right)\right) e^{-\frac{\left(\alpha_{w}-\alpha_{i n s}\left(R_{l, b .}\right)\right]^{2}}{i D_{\alpha}}} e^{i\left[\Theta_{i n s}\left(x^{*}\right)-\Theta_{i n s}\left(x_{l . b .}^{*}\right)+\beta_{w^{*}}^{*} z^{*}-\omega^{*} t^{*}+\phi_{0}\right]}$,

where $R_{h_{0}}$ is the Reynolds number based on the waviness amplitude $h_{0}^{*}$, and

$$
\left.D_{\alpha} \equiv \frac{2}{R_{c l . b .}} \frac{d \alpha_{i n s}}{d x}\right|_{x=1}
$$

is the desynchronization factor, which is related to the chordwise rate of change in the dimensional streamwise wavenumber of the instability mode under consideration. All reference scales used for nondimensionalization purposes in Eqs. (2.18b) and (2.18c) (as well as Eqs. (2.18d) and (2.19) below) are the same as those considered in the localized analysis above, with the assumption that the latter distribution is centered on the chordwise station that corresponds to the lower branch (denoted by the subscript l.b. in these equations).

The factor $D_{\alpha}$ determines the rate at which the surface waviness becomes desynchronized with respect to the instability wave phase $\Theta_{i n s}$ as one moves away from the lower branch location. By 
virtue of its small magnitude, which is related to the ratio of the instability wavelength to the distance from the leading edge to the lower branch location, this factor characterizes the large response of the boundary layer in the presence of distributed roughness, and the strong selectivity of this response in the chordwise wavenumber $\left(\alpha_{w}\right)$ space. In particular, the ratio of the effective vortex amplitude due to a two-dimensional orthogonal lattice of compact roughness shapes to the initial amplitude of the same vortex when it is excited by a single spanwise-periodic array of the same roughness shapes at the lower branch location is given by

$$
\frac{q_{2 D \text { lattice }}^{*}}{q_{1 D \text { array }}^{*}}=\frac{\alpha_{\text {ins }}\left(R_{l . b .}\right)}{\sqrt{i \pi D_{\alpha}}} \exp \left(-\frac{\left[\alpha_{w}-\alpha_{\text {ins }}\left(R_{l . b .}\right)\right]^{2}}{i D_{\alpha}}\right) .
$$

Note that this expression is valid irrespective of the specific shape of the individual roughness element. In fact, the same expression also holds in the corresponding two-dimensional situation, namely the ratio of the effective instability amplitude caused by a series of equidistant roughness strips to the amplitude associated with a single roughness strip.

Now, let us examine the case where the acoustic disturbance is still deterministic and time harmonic, as before, but the roughness-height distribution $h_{w}^{*}\left(x^{*}, z^{*}\right)$ is a random function of both its spatial arguments. For simplicity, we will assume that the latter is also statistically homogeneous in both $x^{*}$ and $z^{*}$, but not necessarily isotropic. The instability motion generated in this case is, again, harmonic in time, but a zero-mean random function of the spatial coordinates $x^{*}$ and $z^{*}$. Then the power spectrum $S_{u}^{*}$ of the amplitude of the chordwise velocity perturbation associated with the generated instability motion is given by

$$
\begin{gathered}
S_{u}^{*}\left(\beta_{w}^{*} ; x^{*}\right)=\sqrt{\frac{2 \pi}{-I m\left(D_{\alpha}\right)}}\left|E_{u}\left(Y_{\max }, R_{l . b .}\right) \Lambda_{u}\left(R_{l . b .}, \theta_{a c}\left(x_{l . b .}^{*}\right)\right)\right|^{2} S_{w}\left(\alpha_{i n s}\left(R_{l . b .}\right), \beta_{w}\right) \\
\exp \left(2\left\{N\left(x^{*}\right)-\left[\alpha_{w}-\alpha_{i n s}\left(R_{l . b .}\right)\right]^{2} / i D_{\alpha}\right\}\right) \mathcal{V}_{a c}^{* 2},
\end{gathered}
$$

where $\operatorname{Im}($.$) denotes the imaginary part of a complex quantity, S_{w}$ is the spectral density function that corresponds to the wall-roughness distribution, and $N\left(x^{*}\right) \equiv-\operatorname{Im}\left(\Theta_{\text {ins }}\left(x^{*}\right)-\Theta_{i n s}\left(x_{i . b .}^{*}\right)\right)$ denotes the linear amplification (with respect to the lower branch location) of the unsteady vortex mode with frequency $\omega^{*}$ and spanwise wavenumber $\beta_{w}^{*}$. For a given $\omega^{*}$ and $\beta_{w}^{*}$, this expression is valid for locations that are sufficiently farther downstream of $x^{*}=x_{l . b}^{*}$.

The results (2.18a) and (2.19) above are identical to those obtained by the author in Ref. [46], with the only difference being related to the definition of the desynchronization factor $D_{\alpha}$, which 
in its present form (see Eq. 2.18b) is applicable to an arbitrary nonsimilar boundary layer over an infinite-span swept wing. Because Ref. [46] provides a detailed derivation of these results, and the interpretation and the implications of the same, we have only quoted the final results here. The reader is also urged to refer to the works by Zavol'skii et al [19] and Tumin and Fedorov [48] in this respect. Provided that the quasi-parallel stability theory is valid in the vicinity of the predicted lower branch location for the stationary mode, the equations (2.18a) through (2.18d), and (2.19) can also be applied towards the stationary receptivity problem, following, of course, a replacement of the subscript $u$ in $u_{u}^{*}$ and $\Lambda_{u}$ by $s$, and the velocity scale $\mathcal{V}_{a c}^{*}$ by the free-stream speed $\mathcal{V}^{*}$.

Because the efficiency function $\Lambda_{u}$ has a rather simple dependence on the orientation of the acoustic wave, Eq. (2.19) can be further generalized to the case where, in addition to the roughness-height distribution, the acoustic disturbances in the free stream are also random in nature. The instability motion generated in this case is a random function of both the spatial and temporal coordinates. If one assumes that the acoustic spectrum is statistically stationary and also isotropic (i.e., $\theta_{a c}$ has an equal probability of assuming any value between $[0,2 \pi]$ ) then the power spectrum of the amplitude of the chordwise-velocity perturbation associated with the generated instability motion is given by

$$
\begin{gathered}
S_{u}^{*}\left(\beta_{w}^{*}, \omega^{*} ; x^{*}\right)=\sqrt{\frac{\pi}{-I m\left(2 D_{\alpha}\right)}}\left|E_{u}\left(Y_{m a x}, R_{l . b .}\right) \Lambda_{u}\left(R_{l . b .} ; \theta_{a c}=\theta_{i n s}\left(x_{l . b .}^{*}\right)\right)\right|^{2} \\
S_{w}\left(\alpha_{i n s}\left(R_{l . b .}\right), \beta_{w}\right) S_{a c}^{*}\left(\omega^{*}\right) \exp \left[2 N\left(x^{*}\right)\right],
\end{gathered}
$$

where $\theta_{i n s}=\arctan \left(\beta_{w} / \alpha_{i n s}\right)$ corresponds to the orientation of the instability-wave vector and the function $S_{a c}^{*}\left(\omega^{*}\right)$ denotes the frequency spectrum of the (dimensional) acoustic slip velocity in the vicinity of the lower branch location for the unsteady vortex mode with frequency $\omega^{*}$ and spanwise wavenumber $\beta_{w}^{*}$.

This completes the theoretical discussion that pertains to the problem of receptivity in threedimensional boundary layers. Much of this discussion and the results can be applied quite readily to other receptivity problems in the same category (e.g., acoustic generation of instability waves in the presence of surface suction through arrays of suction holes with an appropriate spacing). In fact, the discussion related to the effects of roughness geometry is also relevant to the control of boundary-layer instabilities by active wave-cancellation techniques. Numerical results based on the application of this theory are given in the following section. 


\section{Numerical Results}

As seen from the Eqs. (2.10b) and (2.14b), the scaled amplitude of a generated instability mode can always be expressed as the product between an efficiency function $\left(\Lambda_{s}\right.$ or $\left.\Lambda_{u}\right)$, which is independent of the roughness geometry, and a geometry factor $\epsilon_{w} \bar{h}_{w}$, that is simply the amplitude of the Fourier harmonic from the roughness distribution which is resonant with the generated vortex mode. This type of decoupling substantially simplifies the parametric study of such receptivity mechanisms. However, the number of parameters on which the efficiency functions $\Lambda_{s}$ and $\Lambda_{u}$ depend is still quite large, particularly in view of the three dimensionality of the flow involved. In particular, for the FSC family of boundary layers, both $\Lambda_{s}$ and $\Lambda_{u}$ depend on the pressure gradient parameter $\beta_{H}$, the local Reynolds number $R$, and the sweep angle $\theta_{s w}$. In addition, the function $\Lambda_{u}$ also depends on the frequency $\omega$ and the local orientation $\theta_{a c}$ of the free-stream acoustic disturbance. A complete parametric study across this multidimensional space would be a rather formidable task. Therefore, in this paper, we will be content with comparing the numerical values of $\Lambda_{s}$ and $\Lambda_{u}$ in a few typical cases and, in addition, elucidating the general effect of the different types of roughness geometries. It will be seen that even within the limited number of cases considered, the efficiency function $\left|\Lambda_{u}\right|$ can exhibit rather complex patterns, due to the multitudinous influences thereupon. Of course, only the major features of its behaviour will be highlighted in the discussion below.

\subsection{A Comparison of the Efficiency Functions for Stationary and Nonstationary Receptivity}

\subsubsection{Neutral Vortex Modes}

To begin, let us assume that the position of the roughness distribution has been held fixed at $R=400$ and examine the magnitudes of $\Lambda_{s}$ and $\Lambda_{u}$ for the different crossflow modes that are predicted to be neutrally stable at this location by the quasi-parallel stability theory. Receptivity in the vicinity of the lower branch location is most important from a practical standpoint; however, the physics of instability modes in most of the unstable region is, in general, typified by the upper branch mode. In that sense, examining this latter limit is helpful as well. We will consider three different values of the pressure gradient parameter: $\beta_{H}=1.0,0.6$, and -0.1 , respectively. The local sweep angle $\theta_{s w}$ will be assumed to be $45^{\circ}$ in each of the three cases. As pointed out by Mack [33], this particular sweep angle corresponds to the maximum crossflow velocity at a given pressure gradient; therefore, one 
may expect the features of crossflow instability and receptivity to be exhibited in the most prominent fashion near this angle. In fact, because of the strong, favorable pressure gradient at $\beta_{H}=1.0$ and $\beta_{H}=0.6$, the local instability of the associated boundary-layer profiles is purely of the crossflow type. On the other hand, due to the adverse pressure gradient at $\beta_{H}=-0.1$, the associated boundary-layer flow is unstable to both streamwise and crossflow instabilities at the location of receptivity. To get an idea about the locus of neutral modes for a typical FSC boundary layer with a favorable and adverse pressure gradient, respectively, along the chordwise direction, the reader may refer to Figs. 13.7 and 13.9 of Mack [33]. An entire neutral surface for the three-dimensional boundary layer along a swept wing has been plotted by Meyer and Kleiser [6].

In Fig. 3a, we have plotted the efficiency-function magnitudes for the neutral modes along the lower branch at the above three pressure gradients, as functions of the frequency parameter $f \equiv \omega / R$. The corresponding plot for the neutral modes along the upper branch at $R=400$ is given in Fig. 3b. In general, for each neutral mode with frequency $f$ and wavenumber vector $\left(\alpha_{i n s}, \beta_{w}\right)$, another neutral mode exists with frequency $-f$ and wavenumber vector $\left(-\alpha_{i n s},-\beta_{w}\right)$. However, the values of the efficiency function for each pair of modes of this type are complex conjugates of each other. Thus, only one of these two families need be considered for the purpose of a parametric study; in our calculations, we have chosen the family of neutral modes that represents an analytic continuation from the stationary neutral modes with positive spanwise wavenumbers. Thus, each curve in Figs. 3a and $3 \mathrm{~b}$ indicates the variation in $\left|\Lambda_{u}\right|$ with $f$ at a fixed value of $\beta_{H}$; the corresponding symbol denotes the magnitude of $\Lambda_{s}$ for the respective stationary mode magnified twenty times so that the stationary data point can be fitted on the same scale as $\Lambda_{u}$. For computing the $\left|\Lambda_{u}\right|$ variation along the frequency axis, we have assumed the local orientation of the acoustic wave to be fixed at $\theta_{\mathrm{ac}}=0^{0}$.

Figures $3 \mathrm{a}$ and $3 \mathrm{~b}$ show that, for the stagnation point boundary layer $\left(\beta_{H}=1.0\right)$, the range of neutral frequencies at $R=400$ extends from nearly $-1.9 \times 10^{-4}$ along the negative $f$ axis to about $2.6 \times 10^{-4}$ on the positive side. At $\beta_{H}=0.6$, this range shrinks somewhat on both sides of the $f$ axis, whereas at $\beta_{H}=-0.1$, it shrinks substantially, but only along the positive $f$ axis. The maximum (positive) neutral frequency at $\beta_{H}=-0.1$ is only about $0.18 \times 10^{-4}$. Moreover, because the local boundary-layer profile along the chordwise direction is unstable at $\beta_{H}=-0.1$, the range of unstable spanwise wavenumbers in this case also includes the $\beta=0$ mode in a significant range of the neutral frequencies. One may observe that, in general, the $\left|\Lambda_{u}\right|$ curve has a somewhat different behavior 
depending on whether the frequency parameter $f$ is positive or negative. The type of local instability also appears to have an influence on the shape of this curve. For instance, along the lower branch of the neutral locus, one finds a $\left|\Lambda_{u}\right|$ curve with a pronounced oscillatory behaviour in the case of $\beta_{H}=-0.1$ (where the local instability is a combination of both streamwise and crossflow modes), in contrast to a nonoscillatory pattern when $\beta_{H}=1.0$ or 0.6 (in which case the local instability is purely of the crossflow type). However, perhaps the most striking feature of the $\left|\Lambda_{u}\right|$ curves along both upper and lower branches is their rapid rise as the frequency parameter tends to zero along both sides of the frequency axis. Of course, the Stokes wave approximation for the acoustic-field signature, which was used to compute these results, also becomes invalid at sufficiently small values of $|f|$. Strictly speaking, this approximation is only valid when the local Strouhal number $S \equiv f R_{c}^{2}$ is $>>1$. However, the numerical results of Ackerberg and Phillips [50] for the two-dimensional Blasius case indicate that the Stokes wave solution is nearly established when $S=2$. If we assume the same to be the case for a FSC boundary layer, then the results in Figs. 3a and $3 b$ can only be expected to be valid for $|f|>0.25 \times 10^{-4}$. Figures $3 \mathrm{a}$ and $3 \mathrm{~b}$ also indicate that the magnitude of the efficiency function, $\Lambda_{u}$, along both the lower and the upper branch is an increasing function of the Hartree parameter $\beta_{H}$. However, Figs. $4 \mathrm{a}$ and $4 \mathrm{~b}$ below will show that after maximizing the value of $\left|\Lambda_{u}\right|$ over all possible acoustic orientations, the above observations is probably only valid in the range of favorable pressure gradients.

Figures $3 \mathrm{a}$ and $3 \mathrm{~b}$ also suggest that the efficiency function $\left|\Lambda_{s}\right|$ along either neutral branch is also an increasing function of the Hartree parameter $\beta_{H}$. Considering that the local Hartree parameter along a swept wing is equal to 1.0 at the attachment line, and decreases thereafter, this would seem to indicate an increased sensitivity of the boundary-layer flow to roughness elements in the vicinity of the attachment line. However, one should also keep in mind that the local sweep angle also changes quite rapidly near this location, and the results of Figs. $3 a$ and $3 b$ do not consider the variation in $\left|\Lambda_{s}\right|$ with $\theta_{s w}$. In view of the magnification involved in plotting the stationary data points, the magnitude of $\Lambda_{s}$ is obviously much smaller than the average magnitude of $\Lambda_{u}$ for $|f|>0.25 \times 10^{-4}$. To consider a specific case, let us examine the ratio of $\left|\Lambda_{s}\right|$ to the value of $\left|\Lambda_{u}\right|$ at some typical frequency, say $f=-1.0 \times 10^{-4}$. As indicated in Table 1, the value of this ratio is close to 4.3 for the lower branch modes for all three pressure gradients. The corresponding ratios for a positive frequency $f=1.0 \times 10^{-4}$ are somewhat larger: close to 8.2 for $\beta_{H}=1.0$ and 11.8 for $\beta_{H}=0.6$. If the geometry factor has comparable values for 
the wavenumbers of both steady and unsteady vortices, then the amplitude of an acoustic disturbance that is propagating along the chordwise direction would still need to be as high as nearly 10 percent of the local free-stream speed, as long as the initial amplitude of the neutral nonstationary vortex is to equal the initial amplitude of the neutral stationary mode. Also note that the values of the efficiency functions along the upper branch are roughly of the same order of magnitude as the corresponding values along the lower branch for both stationary and nonstationary vortices.

The efficiency function $\left|\Lambda_{u}\right|$ above indicates the frequency dependence of the roughness-induced receptivity mechanism, measured in terms of the maximum velocity perturbation along the direction of the local inviscid streamline. Even if one measures the instability-wave amplitude in terms of the maximum of the chordwise velocity perturbation, or the velocity pertubation perpendicular to the wavenumber vector of the instability mode, the shape of the frequency-response curve is found to remain qualitatively similar in the cases examined above. On the other hand, when the perturbation in surface pressure, or the maximum of the velocity perturbation in the spanwise direction, was chosen as the yardstick for measuring the strength of receptivity, the shape of the efficiency function curve was considerably different. However, because the velocity perturbation along the local streamline is usually the dominant component of the perturbation associated with crossflow modes, all the results in this paper will be based on this quantity.

\subsection{Influence of Acoustic-Wave Orientation on the Unsteady Receptivity}

The values of $\left|\Lambda_{u}\right|$ plotted in Figs. 3a and $3 b$ were computed for a fixed orientation of the acoustic disturbance, namely $\theta_{a c}=0$. However, as discussed in the context of Eq. (2.15), the magnitude of the efficiency function for a given unsteady mode is maximum when the free-stream velocity fluctuation is aligned with the direction of the wavenumber vector associated with this vortex mode. In Figs. 4a and $4 \mathrm{~b}$, we have plotted these maximum values of $\left|\Lambda_{u}\right|$ along the lower and upper branches of the neutral locus, respectively. According to Eq. (2.15), these values are related to those plotted in Figs. 3a and 3b via the relation

$$
\frac{\Lambda_{u}\left(\theta_{a c}=\theta_{i n s}\right)}{\Lambda_{u}\left(\theta_{a c}=0\right)}=\frac{1}{\cos \theta_{i n s}}
$$

where $\theta_{i n s}$ denotes the orientation of the wavenumber vector $\left(\alpha_{i n s}, \beta_{w}\right)$, as defined following Eq. (2.20). The shape of the $\left|\Lambda_{u}\right|$ curves in Figs. $4 \mathrm{a}$ and $4 \mathrm{~b}$ is significantly different from that in Figs. $3 \mathrm{a}$ and $3 \mathrm{~b}$, with the $\Lambda_{u}$ magnitude for positive frequencies being larger than the $\Lambda_{u}$ values for negative $f$ in most 
parts of the neutral range, except for the low-frequency regime. Moreover, it is seen that the two zeros of the $\left|\Lambda_{u}\right|$ curve along the lower branch at $\beta_{H}=-0.1$ in Fig. 3a are absent from the corresponding curve in Fig. 4a. This is merely because these modes correspond to a purely spanwise wavenumber vector, and hence, could not be excited in the previous case, corresponding to an acoustic wave parallel to the chordwise direction. On other hand, one may note that the (near-zero) minimum in the $\left|\Lambda_{u}\right|$ curves along the upper branch for $\beta_{H}=0.6$, and $\beta_{H}=1.0$ in Fig. $3 \mathrm{~b}$ is also present in each of the corresponding curves of Fig. 4b. It was found that this minimum corresponds to a near cancellation between the respective contributions to the instability motion from the source term in the differential equation (2.12a), and the inhomogeneous boundary condition (2.12b).

A comparison of Figs. 4a,4b with Figs. 3a,3b suggests that the minimum level of free-stream fluctuation, which is necessary to excite an unsteady neutral vortex with the same initial amplitude as the neutral stationary vortex, can be reduced significantly in the cases examined by suitably modifying the orientation of the incident acoustic disturbance. Specifically, for the lower branch mode at $f=$ $-1.0 \times 10^{-4}$, one now needs an acoustic fluctuation of approximately 17 percent at $\beta_{H}=1.0$ and 0.6 , but only about 1 percent in the $\beta_{H}=-0.1$ case (see Table 2 for a detailed comparison of the $\left|\Lambda_{u}\right|$ values with $\left.\left|\Lambda_{s}\right|\right)$. However, even these free-stream levels are still quite high when compared to those encountered in a typical flight environment. Moreover, because the wavenumbers of the two vortices are not drastically different, the differences in geometry factors will probably not change the above numbers significantly.

\subsection{Nonneutral Vortices}

Now let us examine the variation in the magnitude of the efficiency function for the values of $\beta_{w}$ which are between the two neutral values or in the subcritical range below the lower branch wavenumber. A partial motivation behind this examination is, of course, to ensure that the receptivity properties found above for the neutral modes are also characteristic of the overall behavior across the entire band of wavenumbers. However, the generation of growing modes, particularly the stationary ones, is deemed particularly important in the present case. The reasoning behind this is as follows. First, because of the primarily inviscid nature of the crossflow instability, the critical Reynolds number at which the vortex of a given wavelength becomes unstable (according to the quasi-parallel theory) is fairly small. Because of this shrinking in the physical length of the region upstream of the lower branch, the possibility exists that any variations in the surface geometry are concentrated primarily 
in the region downstream, where these vortices are already unstable. The relatively small values of Reynolds number at the lower branch station also suggest that the quasi-parallel theory may become inadequate near this location. Then the only region where the predictions based on this theory can be considered to be reasonably accurate corresponds, again, to locations downstream of the lower branch where these vortices are unstable. In particular, when the thickness of the boundary layer has increased sufficiently to be comparable to the spanwise wavelength of the vortex, then the evolution of the latter has been shown to be governed by the stationary form of Rayleigh's equation (Gregory, Stuart and Walker [51], Hall [52]), which demonstrates the basic validity of quasi-parallel results in this regime. Similarly, the nonstationary modes of successively larger frequencies are described asymptotically by the triple-deck (Stewart and Smith [53]), quintuple-deck (Bassom and Gajjar [54]), or the unsteadyRayleigh framework. Because the quasi-parallel theory is uniformly valid (up to the leading order, at least) in all these regimes, the basic validity of the results pertaining to nonstationary receptivity is also believed to be assured in the majority of the frequency domain.

In Figs. 5a through 5c, we have plotted the magnitude of $\Lambda_{u}$ for $\theta_{a c}=0$ at a few selected values of the frequency parameter $f$, along with the magnitude of $\Lambda_{s}$ in the $f=0$ case, for each of the three different pressure gradients under consideration. The wavenumbers corresponding to upper and lower neutral branches have been highlighted by open and filled circles, respectively. The behavior of $\left|\Lambda_{u}\right|$ across the unstable range of wavenumbers is rather different, depending on whether these modes are of pure crossflow type $\left(\beta_{H}=1.0,0.6\right)$, or represent a combination of the crossflow and streamwise instability, as in the $\beta_{H}=-0.1$ case. The variation in $\left|\Lambda_{u}\right|$ is nearly monotonic in the former case, although it can either increase or decrease with $\beta_{w}$, depending, apparently, on whether or not $f$ is positive. In contrast, the $\left|\Lambda_{u}\right|$ curves at $\beta_{H}=-0.1$ exhibit a rather complicated pattern across the two neutral wavenumbers, which involves local minima and maxima. The latter pattern is also observed at $\beta_{H}=1.0$ and at $\beta_{H}=\mathbf{0 . 6}$, but only in the subcritical range of spanwise wavenumbers.

The major noteworthy features of the $\left|\Lambda_{s}\right|$ curves in Figs. $5 \mathrm{a}$ through $5 \mathrm{c}$ are: (i) the rapid rise in $\left|\Lambda_{s}\right|$ up to nearly the lower branch wavenumber, and (ii) a relatively slow variation across the unstable range of wavenumbers that follows this rise. The shape of the $\left|\Lambda_{s}\right|$ curve at other Reynolds numbers is also roughly similar, with the overall magnitude of the efficiency function decreasing with an increase in the Reynolds number (Fig. 5d). Note that in Fig. 5d (and also in Fig. 5e below), we have assumed the Hartree parameter to be constant while varying the Reynolds number; therefore, the local sweep angle 
$\theta_{s w}$ is also varied suitably with $R$. For the reader's convenience, the abscissa in Fig. $5 \mathrm{~d}$ corresponds to the spanwise wavenumber $\beta_{w}^{*}$ nondimensionalized by a uniform length scale that corresponds to $R=400$. Note that the overall shape of the $\left|\Lambda_{s}\right|$ curve for the FSC profiles is quite similar to that of the analogous efficiency function in the rotating disk case (see Choudhari and Streett [23]). However, unlike the FSC case above, the efficiency function in the rotating-disk case showed little variation with respect to the local Reynolds number at sufficiently large values of the azimuthal wavenumber.

Figure 5e illustrates the variation with respect to $R$ in the $\left|\Lambda_{u}\right|$ curve for a crossflow mode of fixed physical frequency, which corresponds to $f=1.0 \times 10^{-4}$ at $R=400$. Again, the overall features appear to be relatively unchanged within the Reynolds-number range considered. Thus, overall, the parametric study for the nonneutral vortices also suggests that the previous conclusion concerning the initial amplitudes of stationary ạnd nonstationary vortices will remain unaffected.

\subsection{Influence of Roughness Geometry}

\subsubsection{A Single, Isolated Roughness Element}

After studying the behavior of the efficiency functions in a few specific cases, we now consider the influence of the roughness geometry upon the initial amplitude of the generated crossflow-vortex mode. In practice, roughness distributions over laminar-flow wings can assume a wide variety of forms. However, one specific geometry that is relevant to laminar flow control applications and is easily realizable in a laboratory experiment corresponds to roughness distributions created through different arrangements of circular dots with a fixed height. Radeztsky et al [5] recently carried out an experiment in which they examined the generation of stationary crossflow modes, caused by individual circular dots of varying heights and diameters. The instability motion observed in an experiment typically consists of a rather narrow spectrum of wavenumbers. Therefore, in order to compare the relative effectiveness of different roughness geometries, it is sufficient to compare the respective amplitudes of a single Fourier harmonic corresponding to the dominant crossflow mode.

Let us first consider the receptivity due to an individual, isolated circular dot of nondimensional radius $R$. This corresponds to a normalized height distribution of

$$
h_{w}^{*}(X, Z) / h_{0}^{*}=1 \quad X^{2}+Z^{2}<R^{2}
$$

and

$$
h_{w}^{*}(X, Z) / h_{0}^{*}=0 \quad \text { otherwise, }
$$


where $h_{0}^{*}$ denotes the maximum height over the cross section of the roughness distribution. The twodimensional Fourier transform of this geometry is independent of the wavenumber orientation and is given in terms of the wavenumber magnitude $k$ by

$$
\overline{\bar{h}}_{w}(\alpha, \beta)=R^{2} \frac{J_{1}(k R)}{k R},
$$

where $J_{1}$ denotes the Bessel function of the first kind with order one. To assess the relative effectiveness of circular dots with different diameters in exciting a given vortex mode of an arbitrary wavenumber $k$, we have plotted the magnitude of the normalized quantity

$$
k^{2} \overline{\bar{h}}_{w}(\alpha, \beta)=k R J_{1}(k R)
$$

in Fig. 6 as a function of the dot radius normalized by the vortex wavelength $\lambda_{\text {ins }}=2 \pi / k$. the normalized geometry factor displays a pattern of alternate maxima and minima. The sequence of these minimum values corresponds to the countably infinite zeros of the Bessel function $J_{1}\left(2 \pi R / \lambda_{\text {ins }}\right)$. The values of the local maxima increase monotonically with $R / \lambda_{\text {ins }}$, with an asymptotic behavior proportional to $\left(R / \lambda_{i n s}\right)^{3 / 2}$ as $R / \lambda_{\text {ins }} \rightarrow \infty$. The first maximum is approximately equal to 1.25 and occurs at a radius that corresponds to $R / \lambda_{\text {ins }} \approx 0.3827$. As the radius of the dot is decreased below this value, the magnitude of the geometry factor decreases and approaches zero at a quadratic rate in the $R / \lambda_{\text {ins }} \rightarrow 0$ limit. The quadratic approach to zero for the two-dimensional transform is considerably faster than the corresponding behavior of the one-dimensional Fourier transform of a rectangular roughness strip in the limit of its width going to zero.

One of the significant outcomes of the work by Radeztsky et al [5] was the experimental demonstration of the influence that the receptivity stage can have on the transition Reynolds number in a swept-wing boundary layer. In particular, these investigators measured the variation in the transitionwedge location downstream of a circular roughness dot as a function of the dot diameter normalized by the wavelength of the dominant crossflow mode. Their findings showed that as the dot diameter was decreased below its initial value, the transition location shifted continually downstream, but at a decreasing rate, so that for roughness dots below a critical size $\left(R / \lambda_{\text {ins }} \approx 0.04\right)$, no further movement in the transition location occurred. Although relating the transition location directly to the initial amplitude of the dominant stationary vortex is difficult, the assumption can be made that a downward shift in the transition location corresponds to a smaller initial amplitude of the vortex. The largest size dot used in the experiments of Radeztsky et al had a diameter of about 0.46 times the wavelength of 
the vortex, or a radius that corresponds to $R / \lambda_{\text {ins }}=0.23$. Because this value is well below the location of the first maximum in Fig. 6, the observations of Radeztsky et al are consistent with the theoretical predictions above.

Similarly, the rapid drop in the magnitude of the geometry factor as $R / \lambda_{\text {ins }} \rightarrow 0$ can explain the observed saturation in the transition location as the dot diameter is reduced below approximately 8 percent of $\lambda_{\text {ins }}$. As seen from Fig. 6 , at $R / \lambda_{\text {ins }}=0.04$, the magnitude of the geometry factor is already about 40 times smaller than its maximum value at $R / \lambda_{\text {ins }} \approx 0.38274$, or about 25 times smaller than its value at $R / \lambda_{\text {ins }}=0.23$, the latter being the maximum dot size used in the above experiments. In short, there is a nonzero roughness-induced receptivity even as the dot size is reduced below the critical value found in the experiments; however, this receptivity is rather weak, and most likely, negligible when compared to the receptivity via other possible mechanisms, such as those mentioned in the introduction. This, we believe, is the reason behind the saturation in the transition location as the dot size is decreased. Radeztsky et al, however, seem to suggest that a smaller roughness simply does not excite the dominant vortex mode, because it cannot introduce any streamwise vorticity at the length scale of the latter.

\subsubsection{A Periodic Array of Compact Roughness Elements}

A single circular dot excites a continuous spectrum of crossflow vortices in the boundary layer, but a periodic array of such dots will only excite a discrete spectrum that corresponds to the different harmonics of the fundamental wavenumber. Given the efficiency function data in Figs. 3 through 5 , the typical initial amplitudes of both stationary and nonstationary vortices excited by a spanwiseperiodic array of these circular dots will be of interest. The fundamental wavenumber of the array can be assumed to be equal to the spanwise wavenumber of the vortex mode under consideration. For simplicity, we will also assume that the radius of each dot corresponds to the first maximum in Fig. 6 (i.e., $R / \lambda_{\text {ins }} \approx 0.383$ ). The magnitude of the geometry factor in this case is given by

$$
\left|\bar{h}_{w}\right|=0.498 \frac{\beta_{w}}{k^{2}}
$$

If we now assume that the nondimensional height $\epsilon_{w}$ of the dots is equal to 0.1 (i.e., $R_{h_{0}}=\mathcal{V}^{*} h_{0}^{*} / \nu^{*}=40$ in this case), then the initial amplitude of the stationary vortex (measured in terms of the maximum velocity perturbation along the direction of the local inviscid streamline) is about 0.57 percent of the free-stream speed for the lower branch mode at $R=400$ and $\beta_{H}=0.6$. If we further assume the 
acoustic-amplitude parameter $\epsilon_{a c}$ to be approximately 0.1 percent, then the maximum amplitude of the lower-branch mode at $f=1.0 \times 10^{-4}$ is nearly 0.02 percent.

Next, we study the variation in the initial amplitude of a neutral instability mode when the orientation of the roughness array is changed from the spanwise direction (with a simultaneous change in the spacing between the adjacent elements to excite the same instability mode in all cases). As shown by Eq. (2.16), the ratio of the instability-wave amplitude in this case to the amplitude caused by a spanwise-periodic array is given by the product of the corresponding ratios of the respective geometry factors and the efficiency functions, respectively. The magnitudes of the latter two ratios, as well of the product thereof, are plotted in Figs. $7 \mathrm{a}$ through $7 \mathrm{c}$ as functions of the array orientation $\theta_{r}$, for a few selected modes along the lower neutral branch at $R=400$ and $\beta_{H}=0.6$. The ratio of the geometry factors essentially exhibits a cosine variation in $\theta_{r}$, with a zero value for roughness arrays aligned with the axis of the vortex mode. Because the latter direction is usually close to that of the local inviscid streamline, one would expect the roughness arrays that are closely aligned with the local free stream to generally produce weaker receptivity. However, the array orientation that corresponds to the maximum of the efficiency-function ratio, which coincides with the direction of the group-velocity vector, also happens to be reasonably close to the direction of the local inviscid streamline. As a result, one observes a rather rapid variation in the instability-wave amplitude when the array direction is varied across $\theta_{r}=\theta_{s w}-\pi / 2($ Fig. $7 \mathrm{c}$ ).

\subsubsection{Nonlocalized Roughness Distributions}

We now examine the distributed generation of stationary and nonstationary vortices caused by two-dimensional lattices of circular dots. For simplicity, we will assume the lattice geometry to be rectangular and aligned with the coordinate axes $X$ and $Z$. Moreover, in view of the strong tuning required for distributed receptivity, we will also assume that the fundamental wavenumber of this distribution along the chordwise direction is nearly equal to the lower branch wavenumber $\alpha_{i n s}$ for a crossflow mode at the specified physical frequency $\omega^{*}$ and the spanwise wavenumber $\beta_{w}^{*}$ (equal to the fundamental wavenumber of the lattice in the $Z$ direction). As seen from Eqs. (2.18) through (2.20), the main factor that determines the effective initial amplitude of the instability wave in the distributedgeometry case is the desynchronization factor $D_{\alpha}$. We have plotted the real and imaginary parts of this factor in Fig. 8 for the crossflow modes along the lower branch at $R=400$ and $\beta_{H}=0.6$. These 
values are significantly higher than the corresponding values in the Blasius case [46]. Substituting the value of $D_{\alpha}$ for the stationary mode into Eq. (2.18d) then suggests that a two-dimensional lattice of compact roughness elements can produce a stationary crossflow vortex that is nearly 3.2 times stronger than the vortex produced by a one-dimensional array of the same roughness elements at $R=400$.

Figure 8 also indicates that, unlike in the Blasius case, the real part of $D_{\alpha}$ is much larger than its imaginary part for at least the particular case examined in the figure. Because the root-mean-' square amplitude of the instability modes induced by a statistically stationary roughness distribution is determined solely by the imaginary part of $D_{\alpha}$, the above finding points towards a strengthened receptivity in that case. Of course, the nonparallel effects could substantially alter the growth rates of the instability mode and, thereby, $\operatorname{Im}\left(D_{\alpha}\right)$ as well, particularly for the lower Reynolds numbers like $R=400$. An investigation related to the effects of curvature and nonparallelism on the stability of three-dimensional boundary layers was recently given by Malik and Balakumar [55].

\section{Summary and Concluding Remarks}

A nonasymptotic theory, which is based on the earlier asymptotic work by Goldstein [17] and Ruban [18] and similar nonasymptotic work by Zavol'skii et al [19], was applied toward the prediction and comparison of the roughness-induced generation of stationary and nonstationary modes of instability in three-dimensional, incompressible boundary layers. A general description of this theory was initially given in Ref. [23], where the theory was utilized in a comparison of the roughness-induced generation of stationary vortices in a rotating-disk boundary layer, with the receptivity of the same flow in a localized region of suction through the disk surface. The nonstationary receptivity via these mechanisms was conjectured to be significantly weaker, since it requires an interaction between the stationary surface disturbance with an unsteady disturbance in the local free stream, and the latter usually has a very small magnitude. The limited parametric study presented in the current paper, which covers the generation of both stationary and nonstationary modes in the FSC family of boundary layers, supports the above conjecture. Although the efficiency function for nonstationary receptivity was found to be much larger (in terms of its average magnitude) that the corresponding function for the stationary receptivity, their ratio is not so large as to compensate for the small unsteady-forcing amplitude. For instance, in the cases we examined at $\beta_{H}=0.6$ and $R=400$, the initial amplitudes of the stationary vortices were estimated to be nearly 20 to 200 times larger than those of the nonstationary vortices, if the local 
acoustic perturbation is assumed to be approximately 0.1 percent of the local free-stream speed. Of course, the smaller initial amplitudes of the nonstationary vortices can be more than compensated for by the their larger (linear) amplification ratios. However, it is also possible that because of their initial lead, and/or their particularly strong influence on the secondary-instability stage (see the work of Kohama et al [4]), the stationary vortices will lead to transition in a swept-wing boundary layer.

Upon completion of this study, we have learned of the recent work by Crouch [56], who has examined the roughness-induced receptivity in a specific case of the FSC family of boundary layers. The basic approach utilized therein is the same as that described previously by Choudhari and Streett in Ref. [23], in the context of a generalization of the Goldstein-Ruban theory for the prediction of crossflowmode generation in the general class of three-dimensional boundary layers. The results presented in Ref. [56] provide additional support to the abovementioned conjecture in Ref. [23].

The boundary-layer flow over two-dimensional surface obstacles of varying length scales was studied using asymptotic methods by Smith, Brighton, Jackson, and Hunt [57]. On the basis of their work, the following physical explanation can be offerred for the roughness-induced generation of stationary crossflow vortices that have wavelengths comparable to the boundary-layer thickness. As shown in Ref. [57], the strongest disturbance due to a surface obstacle of this type is localized to a thin layer immediately adjacent to the surface. Due to the strong viscous action within this layer, the spatially nonuniform displacement of the flow (by the rough surface) is converted into a relatively weaker (specifically, of relative $O\left(R^{-1 / 3}\right)$ ), but commensurately nonuniform, outflux from the outer edge of this thin layer. This outflux, then, drives the disturbance motion inside the main part of the boundary layer which controls the predominantly inviscid crossflow instabilities. Basically, the variation in the sublayer outflux along the crossflow direction acts as a direct source of streamwise vorticity into the main region, thereby exciting the stationary crossflow mode as part of the total mean-flow perturbation. The asymptotic scalings presented in Smith et al would suggest that the initial amplitude of this vortex mode is $O\left(R^{-1 / 3} \epsilon_{w}\right)$, i.e., $\left|\Lambda_{s}\right|=O\left(R^{-1 / 3}\right)$. However, one may recall at this point that the strength of stationary receptivity is related to the component of the base-flow shear $\left(U_{0}^{\prime}(0), W_{0}^{\prime}(0)\right)$ along the direction of the wavenumber vector corresponding to the stationary crossflow mode (see Eq. (2.7c)) The direction of the base-flow shear is usually within a few degrees of the direction of the local inviscid streamline. On the other hand, the wavenumber vectors of crossflow vortices in swept-wing boundary layers are nearly orthogonal to the potential streamline. This would imply that the magnitude of $\Lambda_{s}$ 
is likely to be smaller (numerically) than just $R^{-1 / 3}$.

We also examined the effects of different types of roughness geometries on a theoretical basis, and also with the aid of a numerically based parametric study. In particular, we examined the influence of the size of a roughness element, the orientation of a periodic roughness array, and the distributed generation of instability modes due to two-dimensional lattices and/or stationary random irregularities. As discussed previously in Ref. [46], the assumption of random irregularities significantly reduces the amount of input required for the estimation of receptivity, since the only necessary specifications in this case are: the mean-square height of the surface roughness, and the type and the integral scale of the autocorrelation function that characterizes the roughness-height distribution. Although, for aerodynamically rough surfaces, even this data does not seem to have been available in the past, the recent work of Radeztsky et al. [5] indicates that the possibility of having such data at the disposal of the designer may be realized in not too distant a future. In our current work, we have also indicated how the randomness of the unsteady disturbances could be accomodated into the Goldstein-Ruban type theory. However, the ability to make predictions of this type is, again, contingent on the availability of similar statistics for the disturbance environment in the free stream.

Because the results herein are based on a nonasymptotic framework, a numerical and/or an experimental verification of them will be highly desirable, particularly for the stationary modes in the vicinity of the lower branch, where the influence of nonparallelism may need to be accounted for. Since the instability amplitudes in the distributed-roughness case are particularly sensitive to the stability properties in this region, nonparallelism would have its largest effect on predictions related to this class of problems, particularly when the roughness distribution is random. The influence of nonparallelism on stationary and nonstationary crossflow disturbances has recently been considered by Malik and Balakumar [55]. They find the overall effect of nonparallelism to be destabilizing, but of a higher order. But, it is not clear whether the same conclusion can also be applied to the lower branch modes. Numerical simulations would, therefore, be helpful in a definite characterization of the nonparallel effects in this region.

Our calculations also point toward an increased efficiency of the roughness-induced receptivity to acoustic disturbances of very small frequencies. However, since the Stokes wave approximation utilized in this paper becomes invalid at these smaller frequencies, a separate investigation devoted specifically to this regime is suggested for the future. Again, experiments or simulations that pertain to realistic 
geometries are needed to clarify this issue further. Finally, the influence of roughness elements with moderately large heights, which produce a nonlinear disturbance to the mean boundary layer, must be examined in the case of both stationary and nonstationary receptivity. So far, this problem has only been considered for the receptivity of a two-dimensional boundary layer, by Bodonyi, Duck, Welch, and Tadjfar [58].

\section{Acknowledgments}

Financial support for this work was provided by the Theoretical Flow Physics Branch at the NASA Langley Research Center, Hampton, VA 23681, under contract number NAS1-19299. The author would like to thank Mr. Dennis Bushnell for insisting upon a specific verification of the conjecture in our earlier work [23] regarding the relative initial amplitudes of stationary and nonstationary vortices. The author would also like to thank Prof. Philip Hall for bringing the work by Manuilovich [24] to his attention. In addition, he acknowledges some useful discussions, related to the topic of this paper, with

Dr. Mujeeb Malik and Dr. Craig Streett of NASA Langley Research Center. Assistance of Dr. Ron Joslin in verifying some stability calculations is also gratefully acknowledged.

\section{References}

[1] Saric, W. S., and Reed, H. L., "Stability and Transition of Three-Dimensional Boundary Layers," Ann. Rev. Fluid Mech., Vol. 21, pp. 429-437, 1989.

[2] Balachandar, S., Streett, C. L., and Malik, M. R., "Secondary Instability in Rotating-Disk Flow," J. Fluid Mech., Vol. 242, pp. 323-348, Sept. 1992.

[3] Dagenhart, J. R., Saric, W. S., Mousseux, M. C., and Stack, J. P., "Crossflow-Vortex Instability and Transition on a 45-Degree Swept Wing," AIAA Paper No. 89-1892, June 1989.

[4] Kohama, Y., Saric, W. S., and Hoos, J. A., "A High-Frequency Instability of Crossflow Vortices that Leads to Transition," in Proc. RAS Conf. Boundary-Layer Transition and Control, Cambridge, UK, Apr. 1991.

[5] Radeztsky, R. H., Jr., Reibert, M.S., Saric, W. S., and Takagi, S., "Role of Micron-Sized Roughness in Transition of Swept-Wing Flows," Bull. Amer. Phys. Soc., Vol. 36, No. 10, p. 2630, 1991. 
[6] Meyer, F. and Kleiser, L., "Numerical Investigation of Transition in 3D Boundary Layers," in Proc. AGARD Symp. on Fluid Dynamics of Three-Dimensional Turbulent Shear Flows and Transition, Oct. 3-6, 1988, Cesme, Turkey.

[7] Reed, H. L., “Numerical Simulation of Swept-Wing Flows," NASA CR-189457, Dec. 1991.

[8] Joslin, R. and Streett, C. L., "Crossflow Disturbance Evolution on Swept Wings Computed by. Spatial DNS," Bull. Amer. Phys. Soc., Vol. 37, No. 8, Nov. 1992.

[9] Gaster, M, “A Simple Device for Preventing Turbulent Contamination on Swept Leading Edges," J. R. Aero. Soc., Vol. 69, p. $788,1965$.

[10] Hall, P., "Görtler Vortices in Growing Boundary Layers: The Leading Edge Receptivity Problem, Linear Growth and the Nonlinear Breakdown Stage," Mathematika, Vol. 3, pp. 151-189, 1990.

[11] Denier, J. P., Hall, P., and Seddougui, S. O., "On the Receptivity Problem for Görtler Vortices: Vortex Motions Induced by Wall Roughness," ICASE Report 90-31, 1990; also appeared as Phil. Trans. R. Soc. Lond. A., Vol. 335, pp. 51-85, 1991.

[12] Bassom, A. P., and Hall, P., Vortex Instabilities in 3D Boundary Layers: The Relationship Between Görtler and Crossflow Vortices. J. Fluid Mech. Vol. 232, pp. 647-680, 1991.

[13] Wilkinson, S. P. and Malik, M. R., "Stability Experiments in the Flow over a Rotating Disk," AIAA J., Vol. 23 (4), p. 558, 1985.

[14] Mack, L. M., “The Wave Pattern Produced by Point Source on a Rotating Disk," AIAA Paper 85-0490, 1985.

[15] Waitz, I. A., and Wilkinson, S. P., "Rotating Disk Transition due to Isolated Roughness with Intense Acoustic Radiation," ALAA Paper 88-3759, 1988.

[16] Goldstein, M. E., "The Evolution of Tollmien-Schlichting Waves Near a Leading Edge," J. Fluid Mech., Vol. 127, pp. 59-81, 1983.

[17] Goldstein, M. E., "Scattering of Acoustic Waves into Tollmien-Schlichting Waves by Small Streamwise Variations in Surface Geometry," J. Fluid Mech., Vol. 154, pp. 509-529, 1985. 
[18] Ruban, A. I., "On the Generation of Tollmien-Schlichting Waves by Sound," Transl. in Fluid Dyn., Vol. 19, pp. 709-16, 1985.

[19] Zavol'skii, N. A., Reutov, V. P., and Ryboushkina, G. V., "Generation of Tollmien-Schlichting Waves via Scattering of Acoustic and Vortex Perturbations in Boundary Layer on Wavy Surface," J. Appl. Mech. Techn. Physics, pp. 79-86, 1983.

[20] Goldstein, M. E., and Hultgren, L. S., "Boundary-Layer Receptivity to Long-Wave Free-Stream Disturbances," Ann. Rev. Fluid Mech., Vol. 21, pp. 137-166, 1989.

[21] Kerschen, E. J., “Boundary Layer Receptivity," AIAA Paper 89-1109, 1989.

[22] Kozlov, V. V., and Ryzhov, O. S., "Receptivity of Boundary Layers: Asymptotic Theory and Experiment," Proc. Roy. Soc. Lond., Vol. A-429, pp. 341-373, 1990.

[23] Choudhari, M., and Streett, C. L., "Boundary Layer Receptivity Phenomena in Three-Dimensional and High-Speed Boundary Layers," AIAA Paper 90-5258, Oct. 1990.

[24] Manuilovich, S. V., "Disturbances of a Three-Dimensional Boundary Layer Generated by Surface Roughness," Fluid Dynamics, pp. 764-769, Mar. 1990.

[25] Müller, B. and Bippes, H., "Experimental Study of Instability Modes in a Three-Dimensional Boundary Layer," in Proc. AGARD Symp. on Fluid Dynamics of Three-Dimensional Turbulent Shear Flows and Transition, Oct. 3-6, 1988, Cesme, Turkey.

[26] Saric, W. S., "Experiments on the Stability of Crossflow Vortices in Swept Wing Flows," AIAA Paper 85-0493, 1985.

[27] Balakumar, P., Hall, P., and Malik, M. R., "On the Receptivity and Nonparallel Stability of Travelling Disturbances in Rotating Disk Flow," Theor. Comp. Fluid Dyn., Vol. 3, No. 3, pp. 125-140, 1991.

[28] Kerschen, E. J., and Choudhari, M., "Boundary Layer Receptivity due to the Interaction of FreeStream Acoustic Waves with Rapid Variations in Wall Suction and Admittance Distributions," Bull. Am. Phys. Soc., 1985; see also: Choudhari, Meelan, "Boundary Layer Receptivity Mechanisms Relevant to Laminar Flow Control," Ph.D. dissertation, University of Arizona, 1990. 
[29] Kerschen, E. J., "Linear and Nonlinear Receptivity to Vortical Free-Stream Disturbances," in Boundary Layer Stability and Transition to Turbulence, ed. Reda et al, FED-Vol. 114, ASME, NY, June 1991.

[30] Choudhari, M., and Streett, C. L., "A Finite Reynolds Number Approach for the Prediction of Boundary Layer Receptivity in Localized Regions," NASA TM 102781, Jan. 1991 (also in Phys. Fluids A, Nov. 1992).

[31] Crouch, J. D., "Initiation of Boundary-Layer Disturbances by Nonlinear Mode Interactions," Boundary Layer Stability and Transition to Turbulence, ed. Reda et al, FED-Vol. 114, ASME, NY, pp. 63-68, Jun. 1991 (also in Phys. Fluids A, Jun. 1992).

[32] Cooke, J. C., "The Boundary Layer of a Class of Infinite Yawed Cylinders," Proc. Cambridge Phil. Soc., Vol. 46, pp. 645-648, 1950.

[33] Mack, L.M., "Boundary Layer Linear Stability Theory," AGARD Report 709, 1984.

[34] Bieler, H. and Dallman, U., "Prediction and Analysis of Primary Instability of a Three-Dimensional Swept Plate Boundary Layer," AIAA Paper 87-1337, 1987.

[35] Fischer, T. M., and Dallman, U., "Primary and Secondary Stability Analysis Applied to the DFVLR-Transition Swept-Plate Experiment," in Proc. AGARD Symp. on Fluid Dynamics of Three-Dimensional Turbulent Shear Flows and Transition, Oct. 3-6, 1988, Cesme, Turkey.

[36] Squire, H. B., "On the Stability for Three-Dimensional Disturbances of Viscous Fluid Flow between Parallel Walls," Proc. Roy. Soc. Lond., Vol. A 142, p. 621, 1933.

[37] Briggs, R. J., Electron-Stream Interactions with Plasmas, MIT Press, Cambridge, Mass., 1964.

[38] Bers, A., "Linear Waves and Instabilities," in Physique des Plasmas, eds. C. DeWitt and J. Peyraud, New York, Gordon and Breach, pp. 117-215, 1975.

[39] P. Huerre, and P. A. Monkewitz, "Local and Global Instabilities in Spatially Developing Flows," Ann. Rev. Fluid Mech., Vol. 22, pp. 473-537, 1990.

[40] Gaster, M., "On the Generation of Spatially Growing Waves in a Boundary Layer," J. Fluid Mech., Vol. 22, pp. 433-441, 1965. 
[41] Tam, C. K. W., "The Excitation of Tollmien-Schlichting Waves in Low Subsonic Boundary Layers by Free-Stream Sound Waves," J. Fluid Mech., Vol. 109, pp. 483-501, 1981.

[42] Lam, H. and Rott, N., "Theory of Linearized Time-Dependent Boundary Layers," Cornell University GSAE Rep. AFOSR TN-60-1100, 1960.

[43] Choudhari, M., and Kerschen, E. J., "Instability Wave Patterns Generated by Interaction of Sound Waves with Three-Dimensional Wall Suction or Roughness," AIAA Paper 90-0119, 1990.

[44] Herbert, T., "Analysis of the Subharmonic Route to Transition in Boundary Layers," ALAA Paper 84-0009, 1984.

[45] Nayfeh, A. H., "Stability of Three-Dimensional Boundary Layers," AIAA Journal, Vol. 18, No. 4, pp. 406-416, 1980.

[46] Choudhari, M., "Boundary-Layer Receptivity due to Distributed Surface Imperfections of a Deterministic or Random Nature," To appear in Theoretical and Computational Fluid Dynamics, Feb. 1993 (see also NASA CR-4439, May 1992).

[47] Gaster, M., "Growth of Disturbances in Both Space and Time," Phys. Fluids, Vol. 23, pp. 723-727, 1968.

[48] Tumin, A. M., and Fedorov, A. V., "Generation of Instability Waves in the Boundary Layers on Vibrating Surfaces," J. Appl. Mech. Techn. Physics, pp. 72-79, 1983.

[49] Crouch, J. D., “Nonlocalized Receptivity of Boundary Layers," J. Fluid Mech., Vol. 244, pp. $567-581,1992$.

[50] Ackerberg, R. C., and Phillips, J. H., "The Unsteady Laminar Boundary Layer on a Semi-infinite Flat Plate due to Small Fluctuations in the Magnitude of the Free-Stream Velocity," J. Fluid Mech., Vol. 51, pp. 137-157, 1972.

[51] Gregory, N., Stuart, J. T., and Walker, W. S. On the Stability of Three Dimensional Boundary Layers with Application to the Flow due to a Rotating Disk," Phil. Trans. R. Soc. Lond. A, Vol. 248, pp. 155-199, 1955. 
[52] Hall, P., "An Asymptotic Investigation of the Stationary Modes of Instability of the Boundary Layer on a Rotating Disk," Proc. Roy. Soc. Lond. A, Vol. 406, p. 93, 1986.

[53] Stewart, P. A., and Smith, F. T., "Three-Dimensional Instability in Steady and Unsteady Nonparallel Boundary Layers, Including Effects of Tollmien-Schlichting Disturbances and Crossflow," Proc. Roy. Soc. Lond., Ser. A, Vol. 409, pp. 229-248, 1988.

[54] Bassom, A. B., and Gajjar, J. S. B., "Nonstationary Crossflow Vortices in Three-Dimensional Boundary Layer Flows," Proc. Roy. Soc. Lond. A, Vol. 417, pp. 179-212, 1988.

[55] Malik, M. R., and Balakumar, P., "Linear Stability of Three-Dimensional Boundary Layers: Effects of Curvature and Non-Parallelism," AIAA Paper 93-0079, 1993.

[56] Crouch, J. F., "Receptivity of Three-Dimensional Boundary Layers," ALAA Paper 93-0074, 1993.

[57] Smith, F. T., Brighton, P. M., Jackson, P. S., and Hunt, J. C. R., "On Boundary Layer Flow Past Two-Dimensional Obstacles," J. Fluid Mech., Vol. 113, pp. 123-152, 1981.

[58] Bodonyi, R. J., Welch, W. J. C., Duck, P. W., and Tadjfar, M., “A Numerical Study of the Interaction Between Unsteady Free-Stream Disturbances and Localized Variations in Surface Geometry," J. Fluid Mech., Vol. 209, pp. 285-308, 1989. 


\section{Table 1}

The ratio $\left|\frac{\Lambda_{u}}{\Lambda_{a}}\right|$ for neutral modes at $R=400$ when $\theta_{a c}=0\left(f_{0} \equiv f \times 10^{6}\right)$

\begin{tabular}{|c||c|c|c|c||c|c|c|c|}
\hline \multicolumn{1}{|c||}{$\beta_{H}$} & \multicolumn{3}{c||}{$f_{0}$ along lower branch } & \multicolumn{4}{c|}{$f_{0}$ along upper branch } \\
\hline & -200 & -100 & 100 & 200 & -200 & -100 & 100 & 200 \\
\hline 1.0 & & 4.2 & 8.2 & 7.8 & & 7.8 & 25.0 & 15.5 \\
\hline 0.6 & & 4.4 & 11.8 & & & 1.05 & 26.9 & \\
\hline-0.1 & 16.0 & 4.2 & & & 4.2 & 7.8 & & \\
\hline
\end{tabular}

\section{Table 2}

The ratio $\left|\frac{\Lambda_{4}}{\Lambda_{0}}\right|$ for neutral modes at $R=400$ when $\theta_{a c}=\theta_{\text {ins }} \quad\left(f_{0} \equiv\right.$ $f \times 10^{6}$ )

\begin{tabular}{|c||c|c|c|c||c|c|c|c|}
\hline \multicolumn{1}{|c||}{$\beta_{H}$} & \multicolumn{3}{c||}{$f_{0}$ along lower branch } & \multicolumn{4}{c|}{$f_{0}$ along upper branch } \\
\hline & -200 & -100 & 100 & 200 & -200 & -100 & 100 & 200 \\
\hline 1.0 & & 5.7 & 24.1 & 33.7 & & 11.4 & 43.0 & 31.5 \\
\hline 0.6 & & 5.9 & 40.7 & & & 1.5 & 46.2 & \\
\hline-0.1 & 34.8 & 100.4 & & & 4.3 & 11.1 & & \\
\hline
\end{tabular}




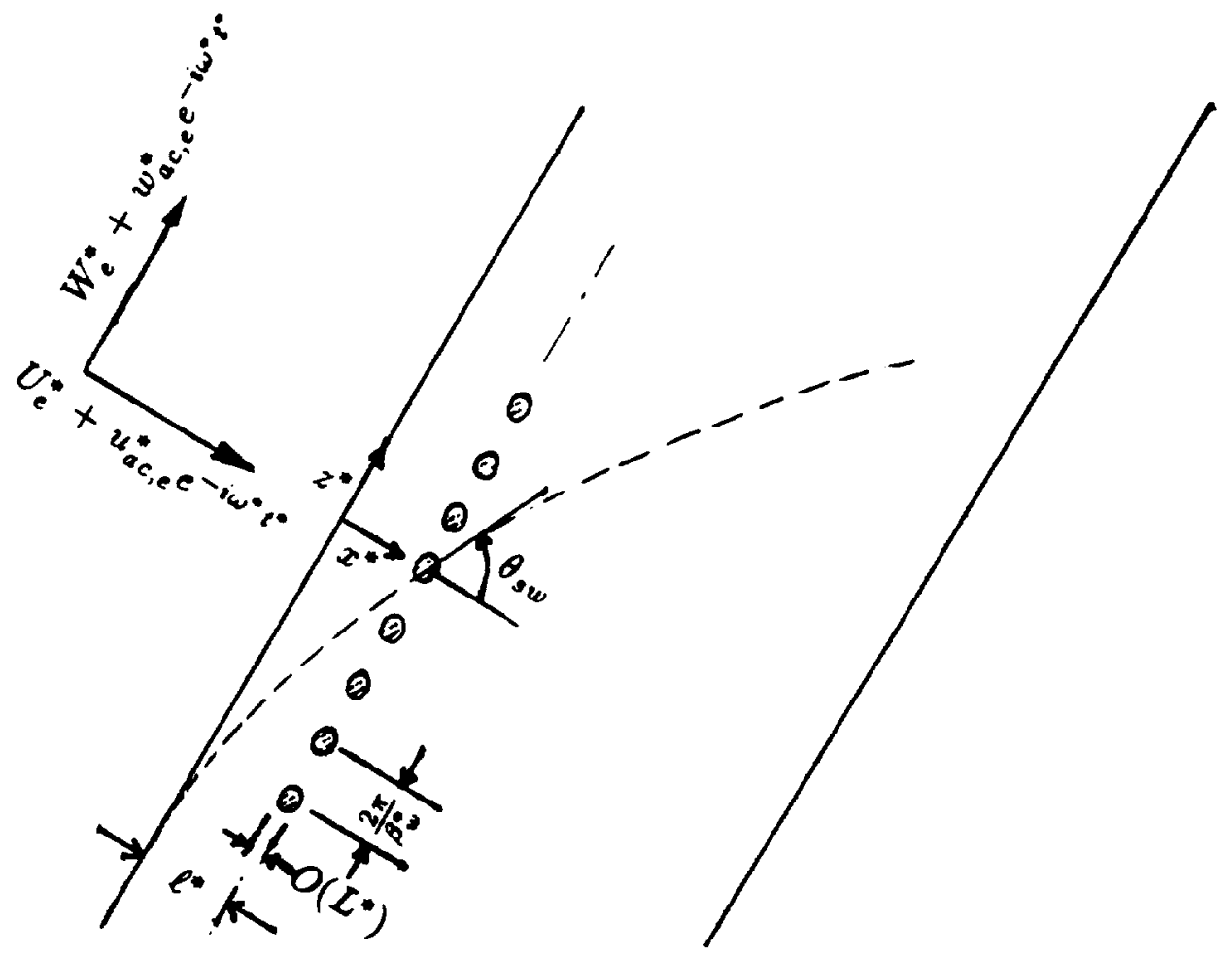

Fig. 1. Schematic of problem geometry. 


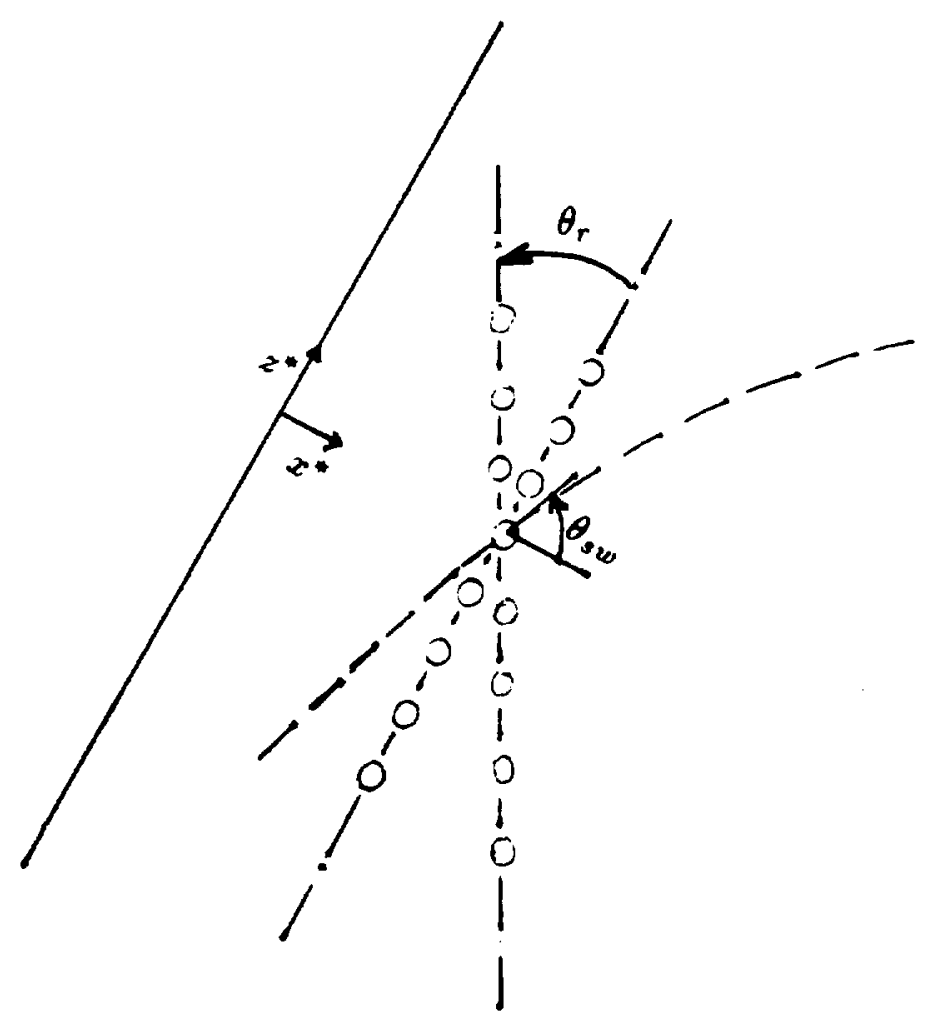

Fig. 2. Receptivity due to skewed roughness arrays. 


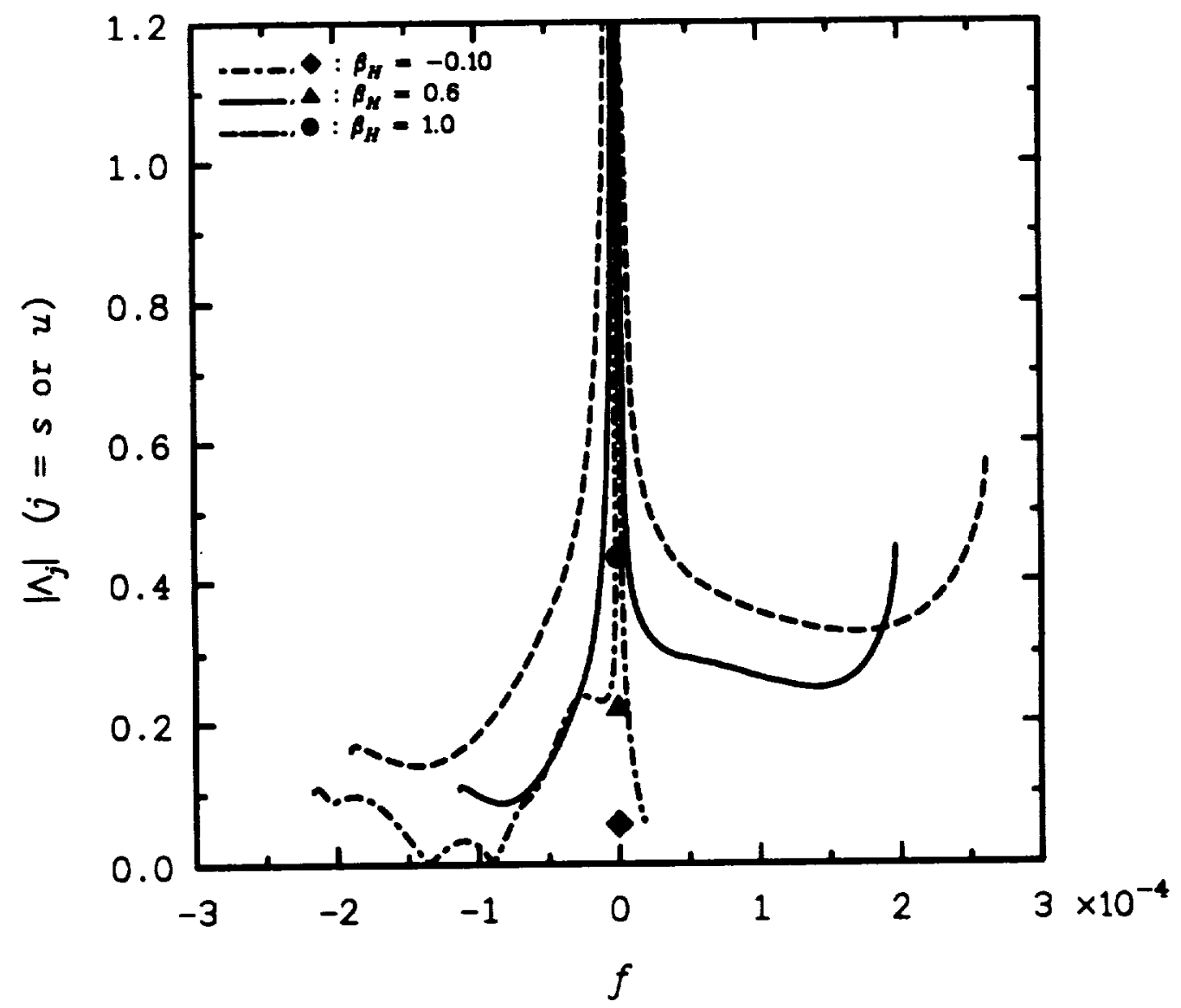

Fig. 3 a. Magnitude of efficiency function along lower branch of neutral curve plotted as function of frequency parameter $f$. Curves indlcate varlation in $h_{w}$. whereas symbols correspond to 10 times $M_{y} \mid\left(R=400, \theta_{s w}=45^{\circ}\right.$, and $\left.\theta_{\alpha c}=0^{\circ}\right)$. 


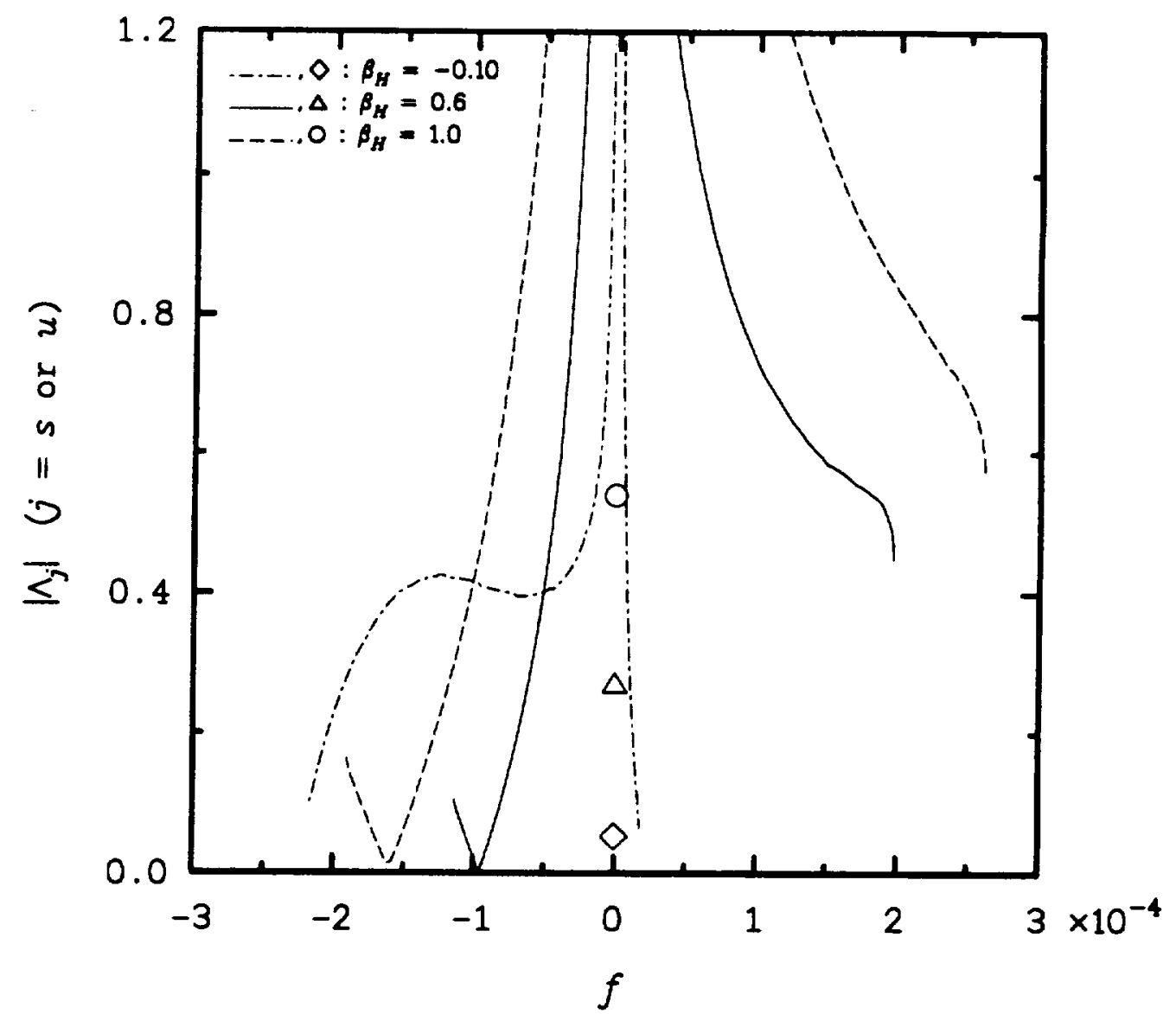

Fig. 3 b. Magnitude of efficiency function along upper branch of neutral curve plotted as function of frequency parameter $f$. Curves indicate varlation in $M_{\mathrm{u}}$. whereas symbols correspond to 10 times $\left|\Lambda_{s}\right|\left(R=400, \theta_{s w}=45^{\circ}\right.$, and $\left.\theta_{a c}=0^{\circ}\right)$. 


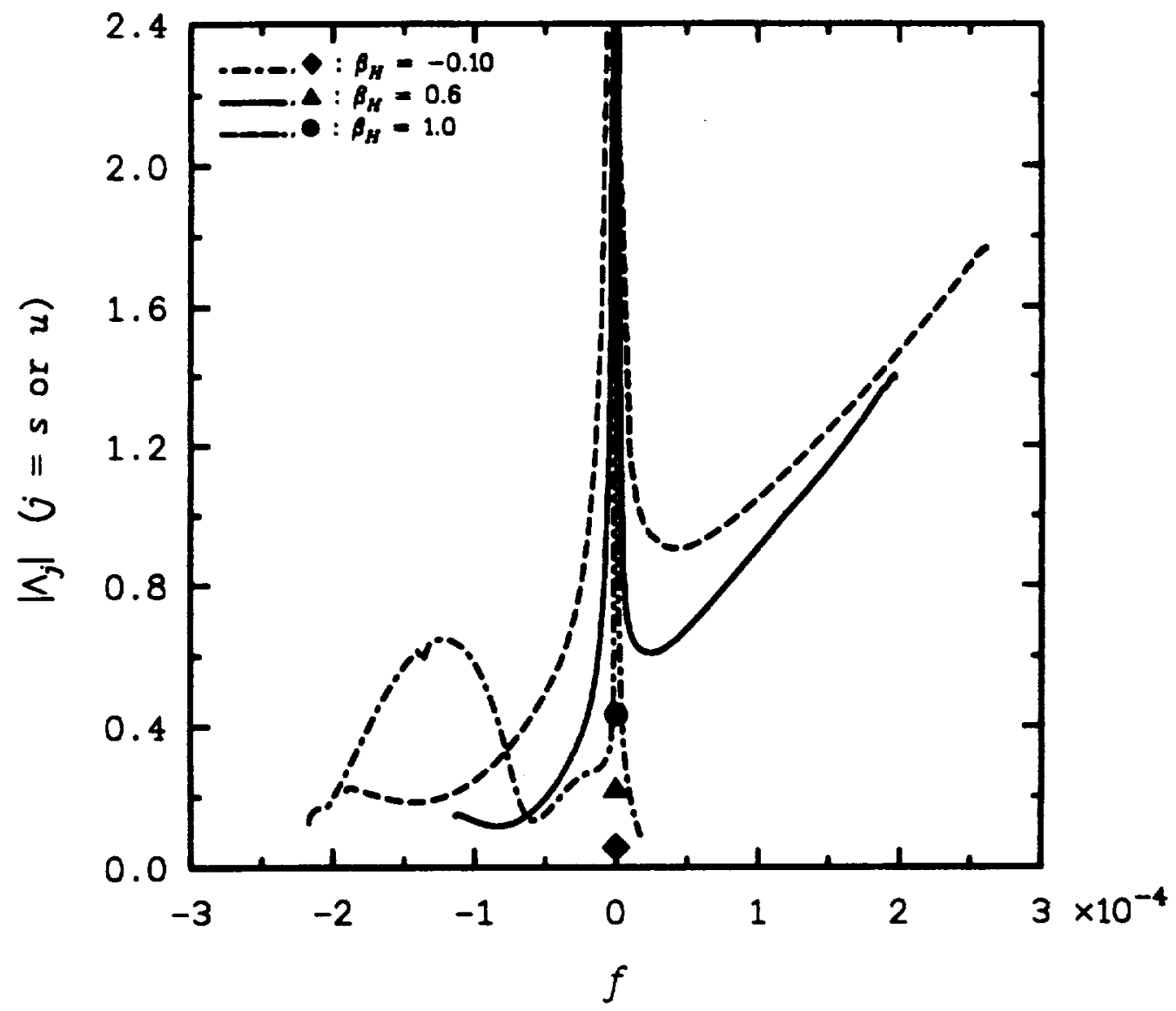

Flg. 4 a. Magnitude of efficiency function along lower branch of neutral curve plotted as function of frequency parameter $f$. Curves indicate varlation in $M_{\mathrm{l}}$. whereas symbols correspond to 10 times $M_{s} \mid\left(R=400, \theta_{s w}=45^{\circ}\right.$, and $\left.\theta_{a c}=\theta_{\text {tns }}\right)$. 


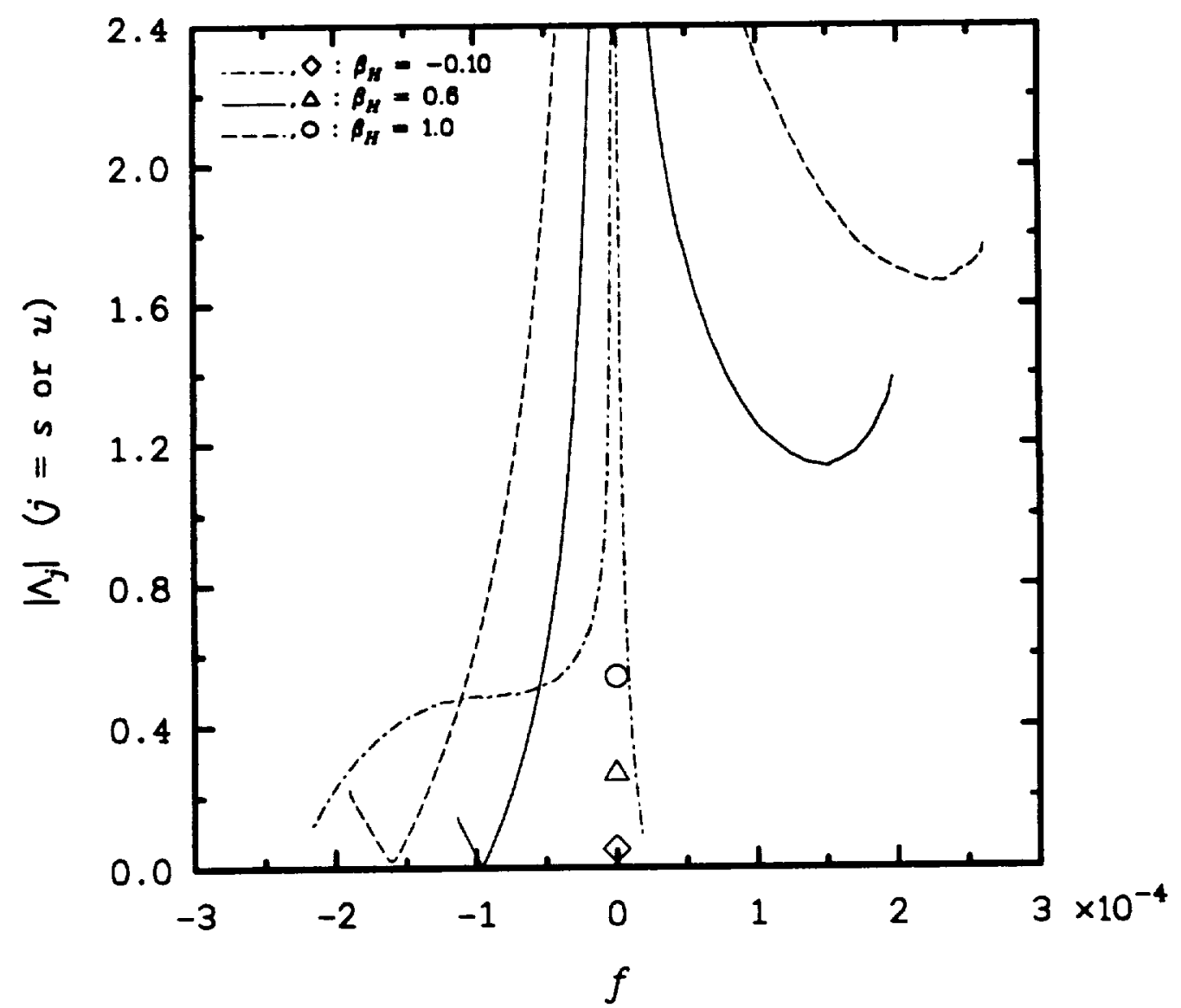

Fig. 4 b. Magnitude of efficiency function along upper branch of neutral curve plotted as function of frequency parameter $f$. Curves indicate varlation in $\left|\Lambda_{W}\right|$, whereas symbols correspond to 10 times $\left|\Lambda_{s}\right|\left(R=400, \theta_{s w}=45^{\circ}\right.$. and $\left.\theta_{\infty e}=\theta_{\text {tne }}\right)$. 


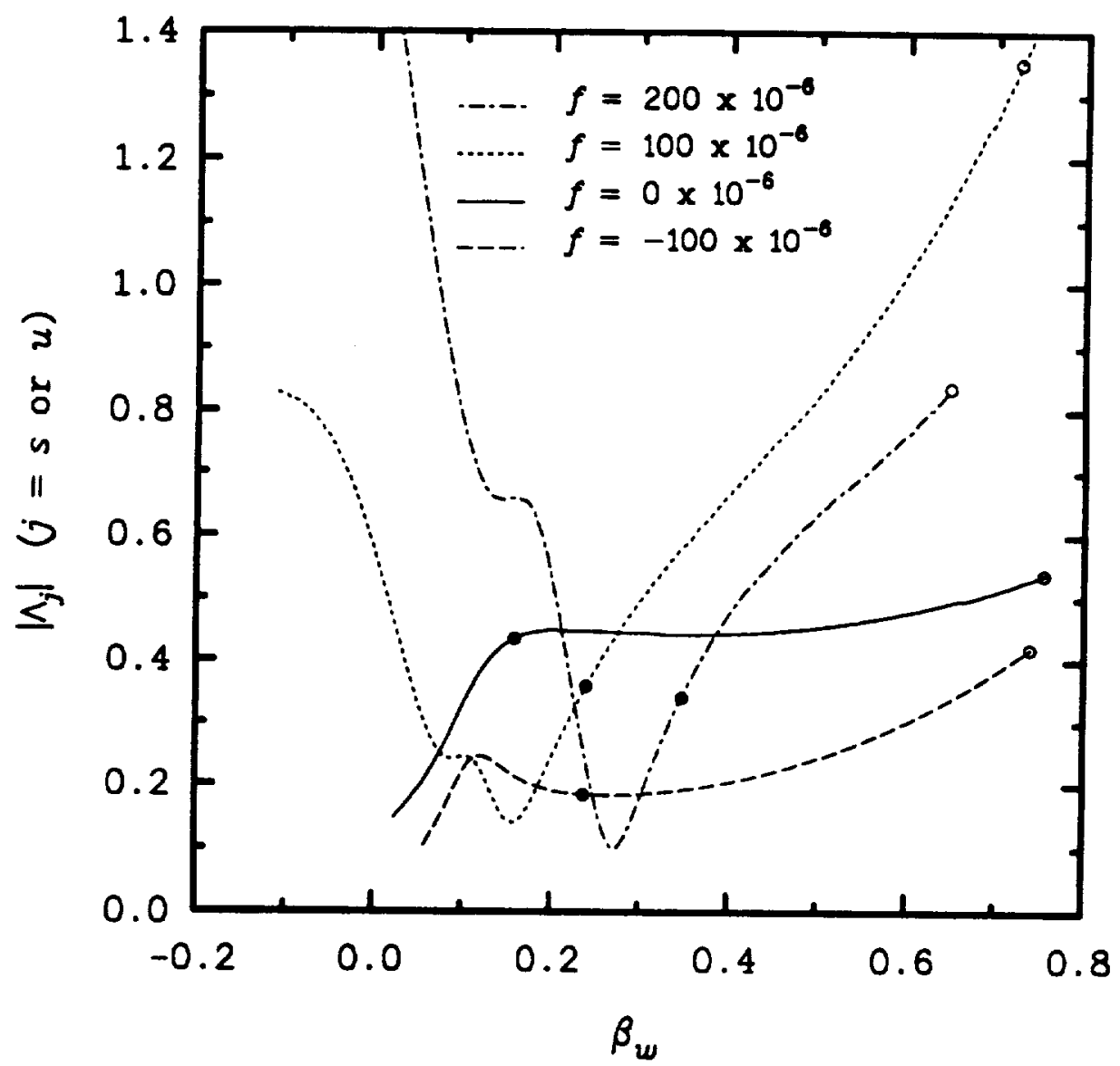

Fig. 5 a. Magnitude of efficiency function for fixed frequency disturbance plotted as function of spanwise vavenumber.

$\boldsymbol{\beta}_{w}$. For $f=0$, efficlency function $\left|\Lambda_{s}\right|$ has been magnified by factor of 10 . Lower- and upper-branch neutral wavenumbers at each frequency are indicated by filled and open clrcles. respectively $\left(\beta_{H}=1.0, R=400, \theta_{s w}=45^{\circ}, \theta_{a c}=0^{\circ}\right)$. 


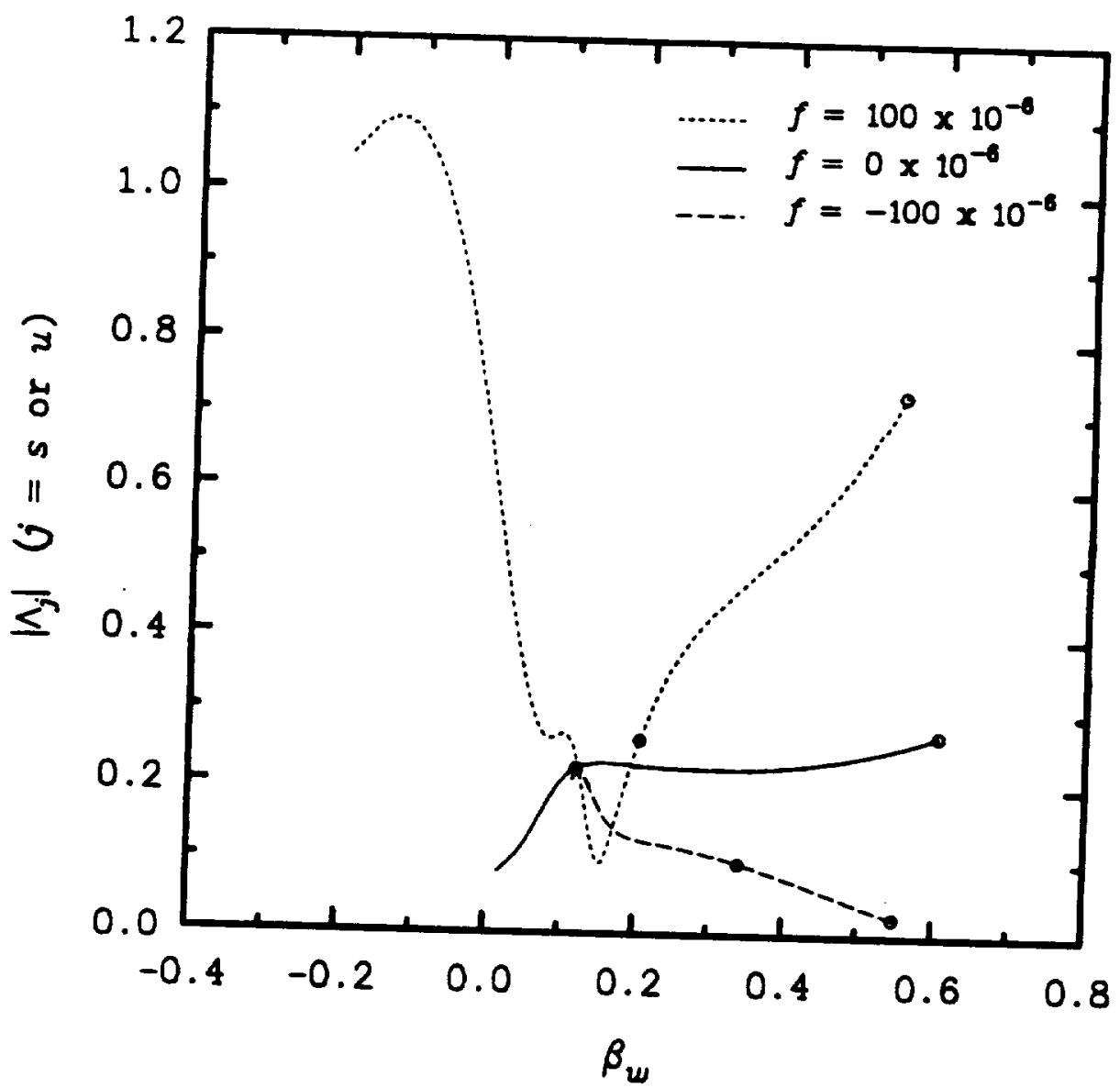

Fig. 5 b. Magnitude of efficlency function for fixed frequency disturbance plotted as function of spanvise vavenumber. $\beta_{w}$. For $f=0$, efflclency function $\mid \Lambda_{d}$ has been magnifled by factor of 10 . Lower- and upper-branch neutral wavenumbers at each frequency are indicated by filled and open circles. respectively $\left(\beta_{H}=0.6, R=400, \theta_{s w}=45^{\circ}, \theta_{a c}=0^{\circ}\right)$. 


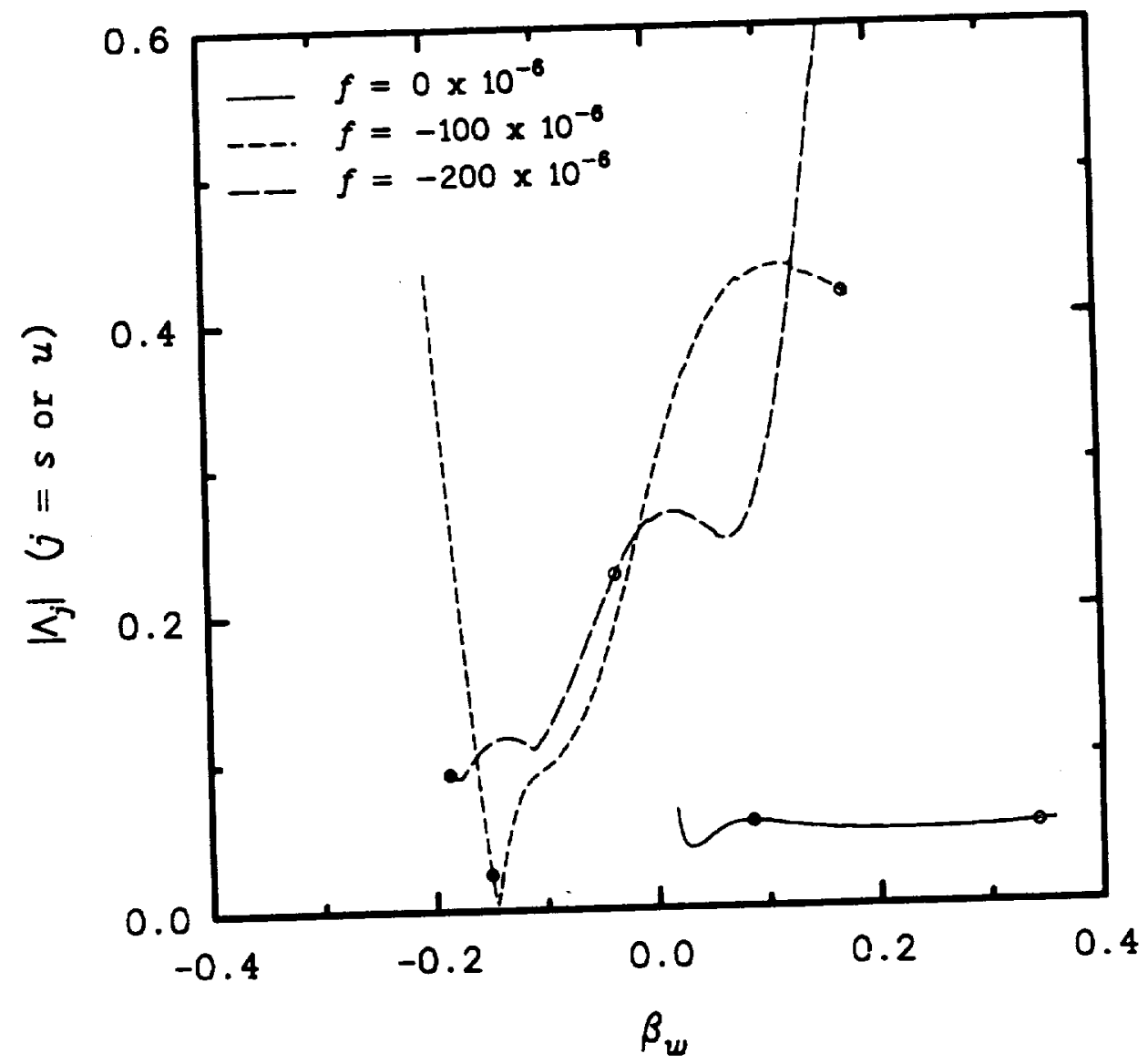

Eig. $5 \mathrm{c}$. Magnitude of efflciency function for fixed frequency disturbance plotted as function of spanvise vavenumber. $\beta_{w}$. For $f=0$, efflclency function $\mu_{s}$ has been magnifled by factor of 10 . Lover- and upper-branch neutral wavenumbers at each frequency are indicated by fllled and open circles. respectively $\left(\beta_{H}=-0.10, R=400, \theta_{s w}=45^{\circ}, \theta_{a c}=0^{\circ}\right)$. 


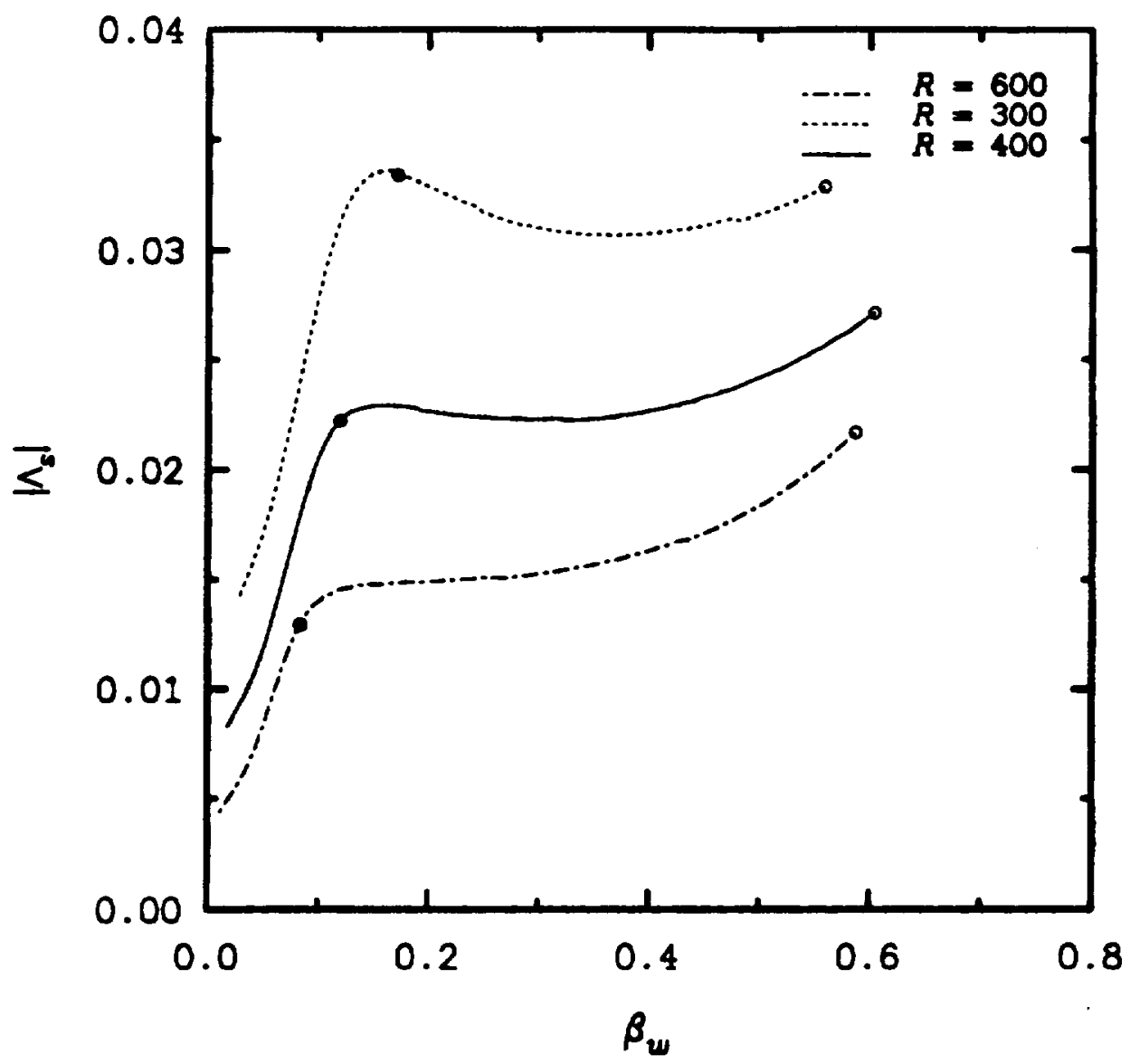

Fig. $5 \mathrm{~d}$. Magnitude of efficiency function $\lambda_{3}$ as function of spanvise vavenumber $\beta_{w}$ with the local Reynolds number $R$ as parameter. For each curve in plot, $\beta_{w}$ has been scaled uniformly by local length scale at $R=400$. 


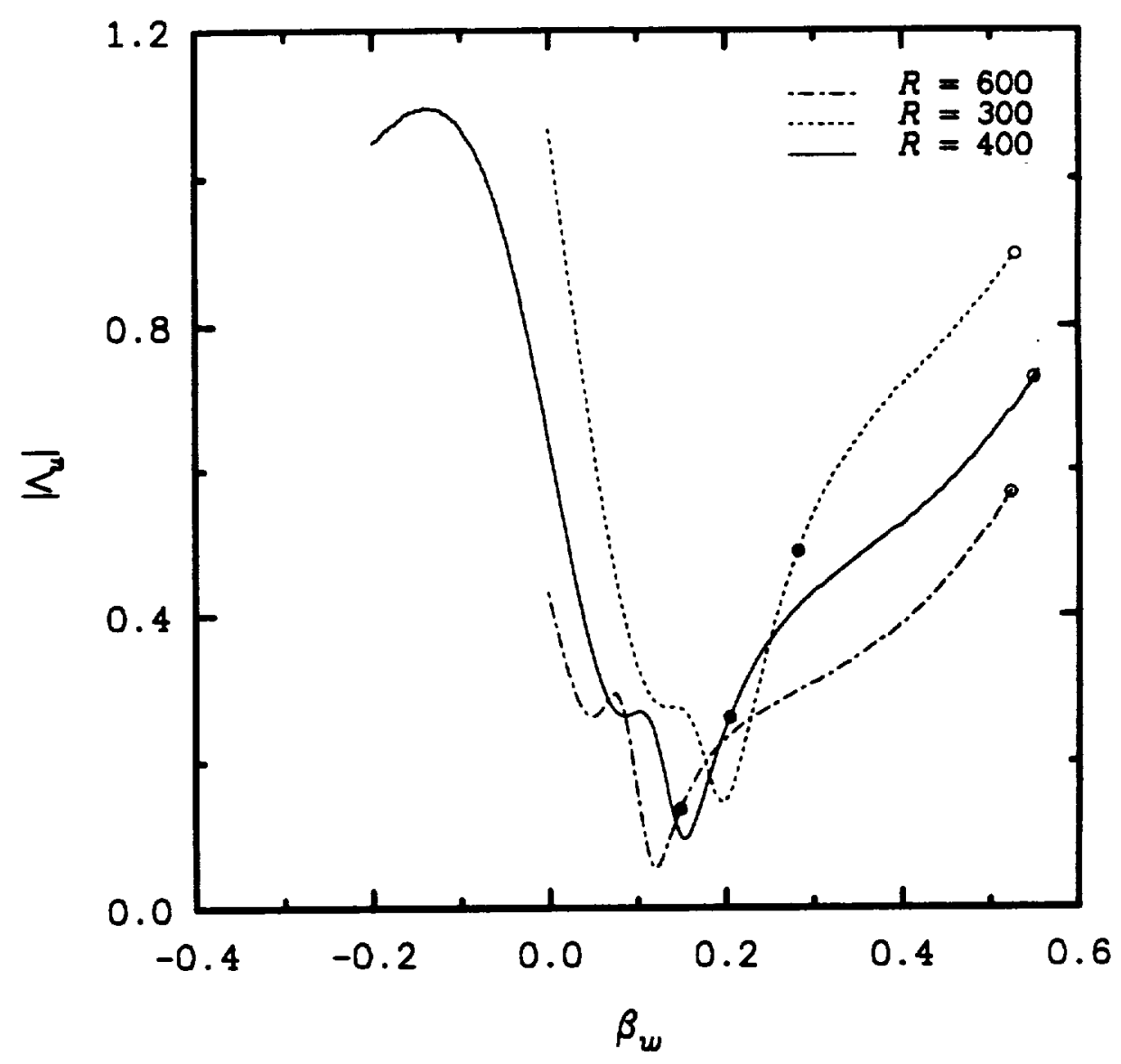

Fig. 5 e. Magnltude of efficiency function $\Lambda_{2}$ for fixed frequency disturbance (corresponding to $f=100 \times 10^{-6}$ at $R=400$ ) as function of spanvise vavenumber $\beta_{w}$ with the local Reynolds number $R$ as parameter. For each curve in plot. $\beta_{w}$ has been scaled uniformly by local length scale at $R=400$. 


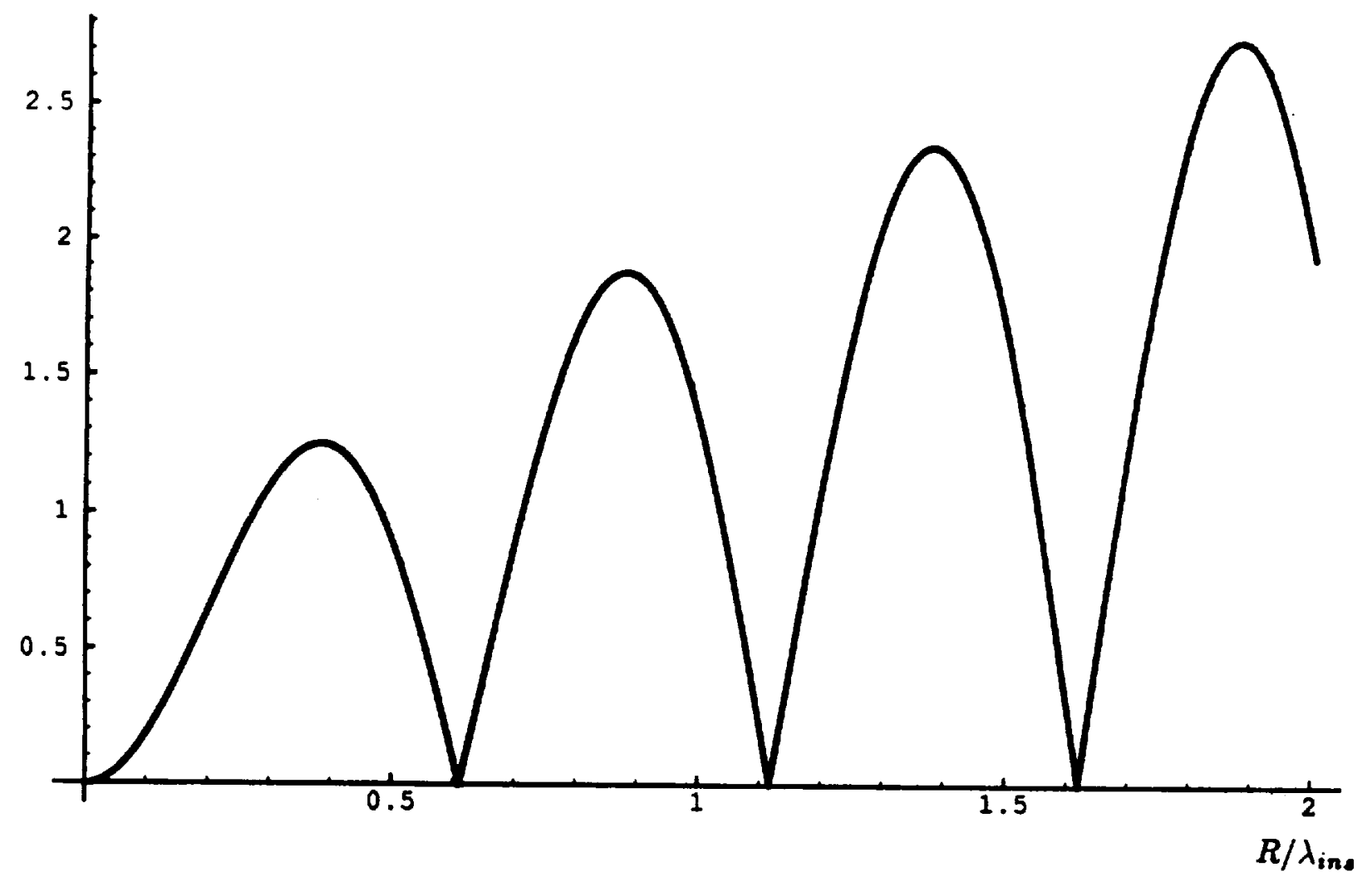

Fig. 6. Influence of dot diameter on magnitude of geometry factor. 


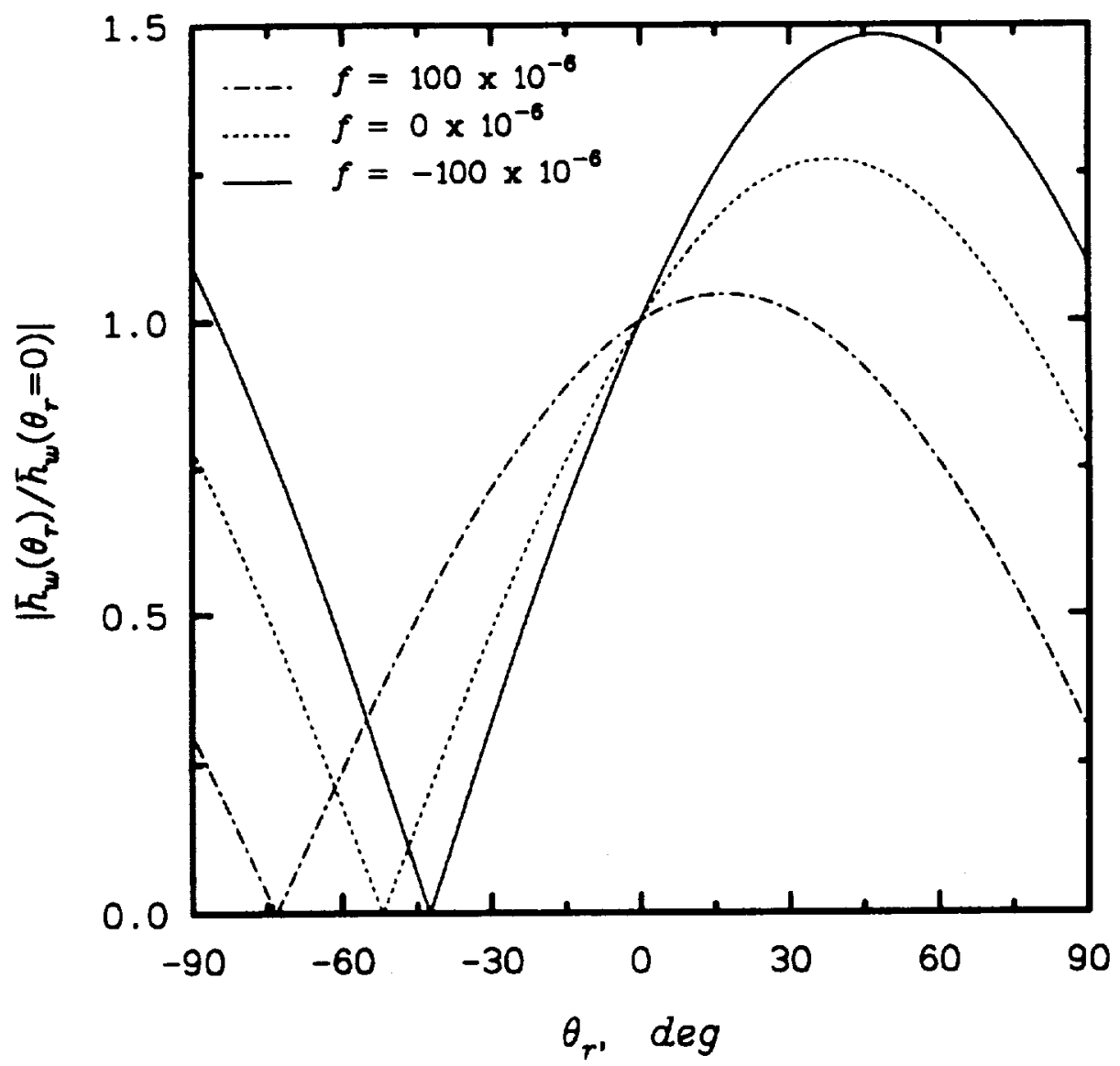

Fig. 7a. $\left|\hbar_{w}\left(\theta_{r}\right) / \hbar_{w}\left(\theta_{r}=0\right)\right|$ plotted as a function of $\theta_{r}$ with nondimensional acoustic frequency $f$ as parameter $\left(\beta_{H}=0.6, R=400, \theta_{s w}=45^{\circ}\right)$. 


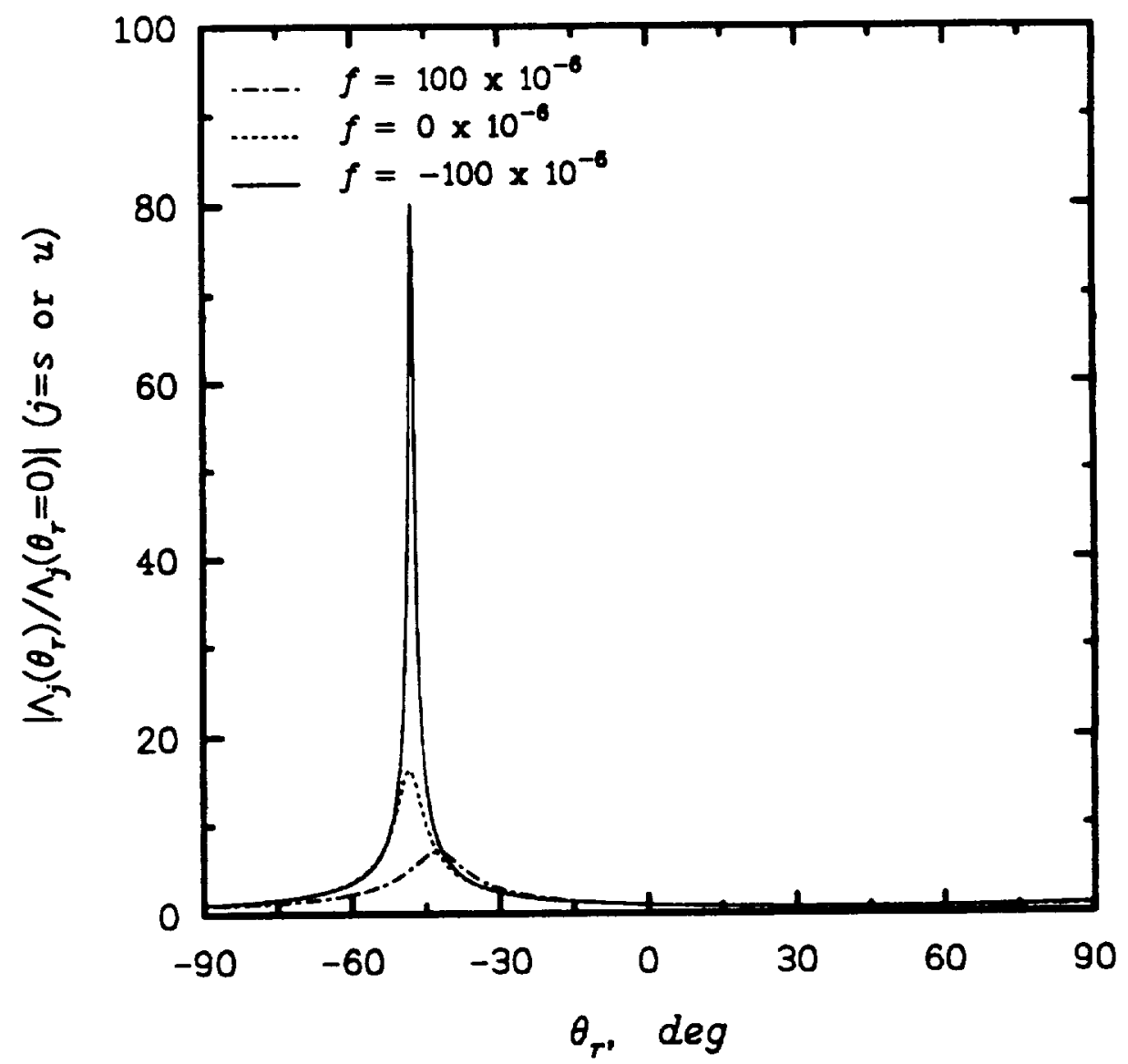

Fig. 7b. $\quad \zeta_{y}\left(\theta_{r}\right) / \Lambda_{y}\left(\theta_{r}=0\right) \mid(j=s$ or $u)$ plotted as a function of $\theta_{r}$ with nondimensional acoustic frequency $f$ as parameter $\left(\beta_{H}=0.6, R=400, \theta_{s w}=45^{\circ}\right)$. 


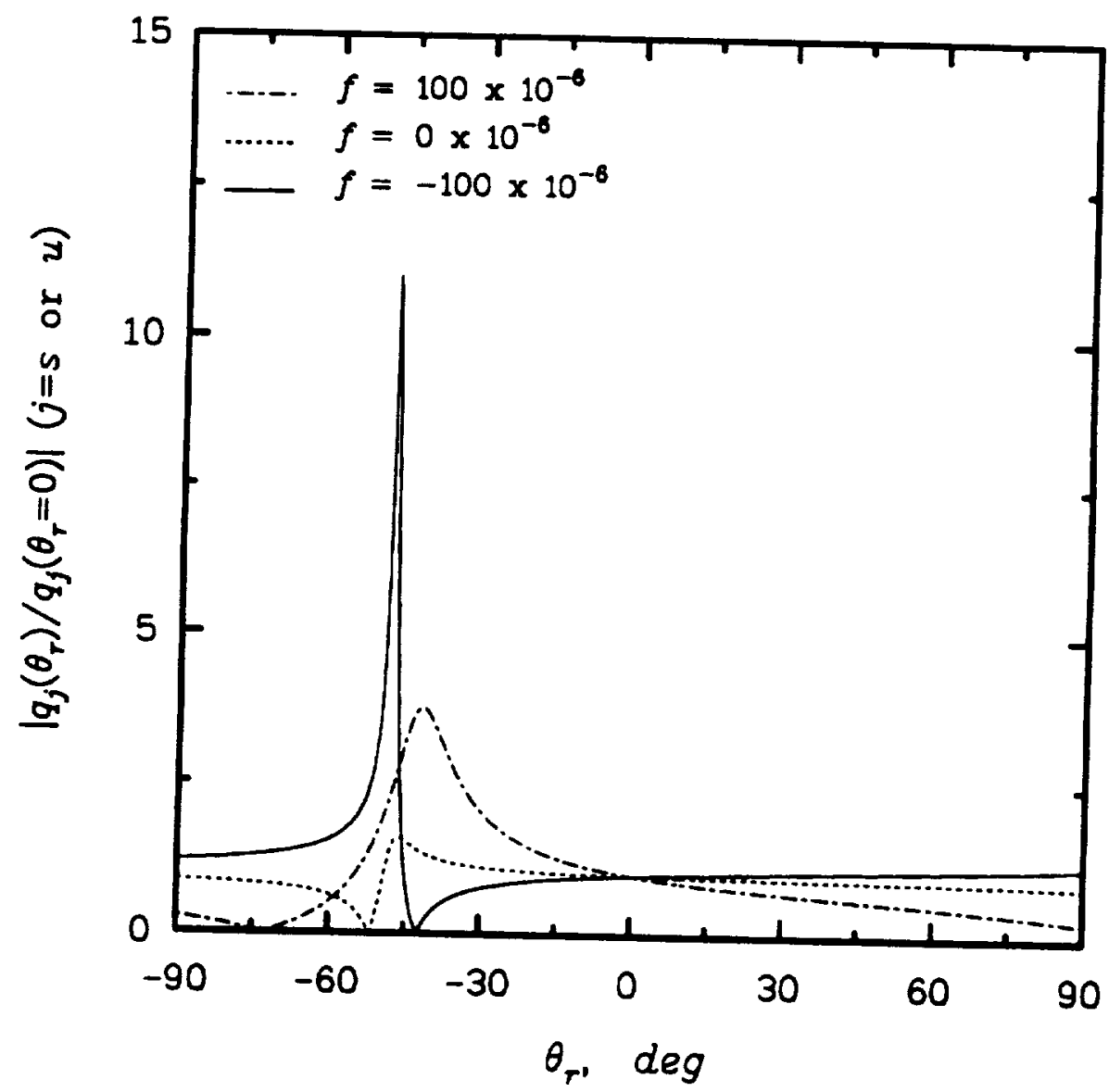

Fig. 7c. $\left|q_{j}\left(\theta_{r}\right) / q_{j}\left(\theta_{r}=0\right)\right|(j=s$ or $u)$ plotted as a function of $\theta_{r}$ with nondimensional acoustic frequency $f$ as parameter $\left(\beta_{H}=0.6, R=400, \theta_{\text {sw }}=45^{\circ}\right)$. 


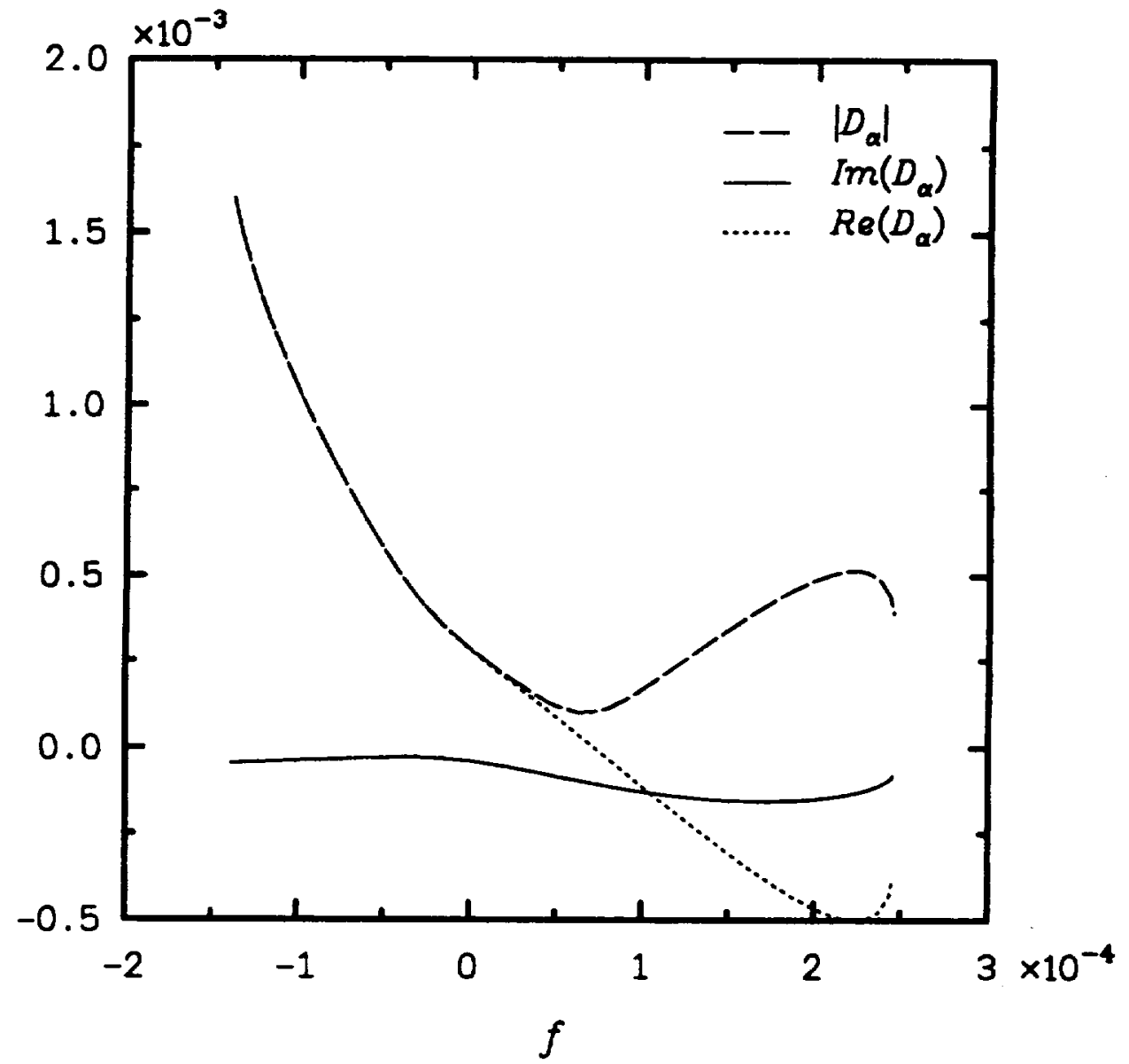

Fig. 8. Variation of desynchronization factor $D_{a}$ with frequency parameter $f$ for lower branch modes at $R=400 . \theta_{\text {sw }}=45^{\circ}\left(\beta_{H}=0.6\right)$. 




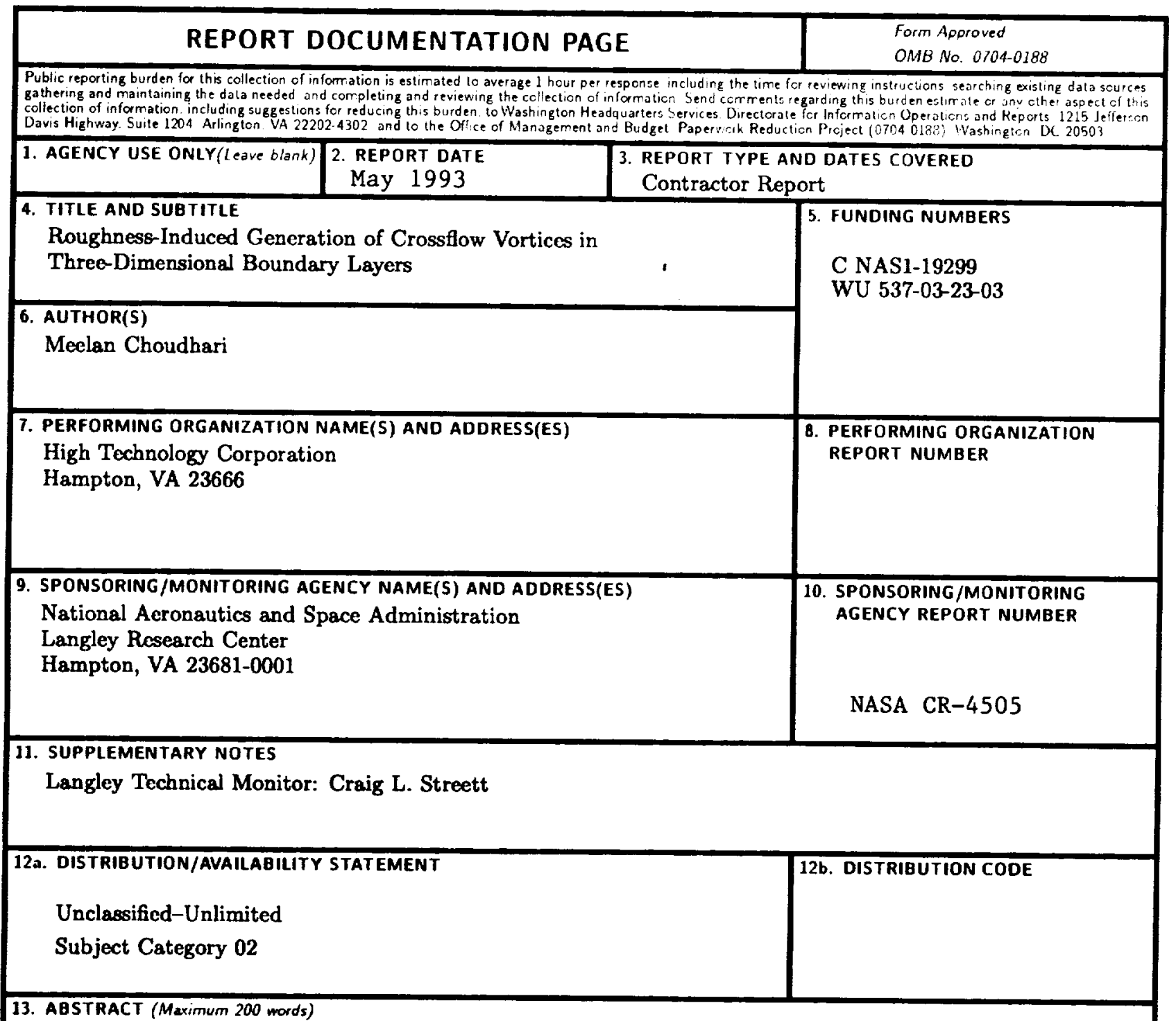

The receptivity theory of Goldstein and Ruban is extended within the nonasymptotic (quasiparallel) framework of Zavol'skii et al to predict the roughness-induced generation of stationary and nonstationary instability waves in three-dimensional, incompressible boundary layers. The influence of acoustic-wave orientation, as well as that of different types of roughness geometries, including isolated roughness elements, periodic arrays and two-dimensional lattices of compact roughness shapes, as well as random, but spatially homogeneous roughness distributions, is examined. The parametric study for the Falkner-Skan-Cooke family of boundary layers supports our earlier conjecture that the initial amplitudes of roughness-induced stationary vortices are likely to be significantly larger than the amplitudes of similarly induced nonstationary vortices in the presence of acoustic disturbances in the free stream. Maximum unsteady receptivity occurs when the acoustic velocity fluctuation is aligned with the wavenumber vector of the unsteady vortex mode. On the other hand, roughness arrays that are oriented somewhere close to the group velocity direction are likely to produce higher instability amplitudes. Limitations of the nonasymptotic theory are discussed, and future work is suggested.

\begin{tabular}{|c|c|c|c|}
\hline \multirow{2}{*}{\multicolumn{3}{|c|}{$\begin{array}{l}\text { 14. SUBJECT TERMS } \\
\text { Threc-dimensional boundary layer; Laminar-turbulent transition; Receptivity; Sur- } \\
\text { face roughness; Crossflow instability }\end{array}$}} & $\begin{array}{l}\text { 15. NUMBER OF PAGES } \\
60\end{array}$ \\
\hline & & & $\begin{array}{r}\text { 16. PRICE CODE } \\
\text { A04 }\end{array}$ \\
\hline $\begin{array}{l}\text { 17. SECURITY CLASSIFICATION } \\
\text { OF REPORT } \\
\text { Unclassified }\end{array}$ & $\begin{array}{l}\text { 18. SECURITY CLASSIFICATION } \\
\text { OF THIS PAGE } \\
\text { Unclassified }\end{array}$ & $\begin{array}{l}\text { 19. SECURITY CLASSIFICATION } \\
\text { OF ABSTRACT } \\
\text { Unclassified }\end{array}$ & $\begin{array}{l}\text { 20. LIMITATION } \\
\text { OF ABSTRACT } \\
\text { UL }\end{array}$ \\
\hline NSW & & & $\begin{array}{l}\text { Standard Form } 298(\text { Rev. } \\
\text { Prescribed by ANSI Std Z39 } 18 \\
\text { 298.102 }\end{array}$ \\
\hline
\end{tabular}

